Journal of Environmental
ASSN: $2525-815 \mathrm{X}$

\title{
Análise fatorial na identificação dos fatores para obtenção de índice climatológico
}

\section{Factor analysis in the identification of factors for the obtainment of climatological index}

\author{
Antonio Ricardo Santos de Andrade ${ }^{\mathrm{a}}$, Edijailson Gonçalves Silva ${ }^{\mathrm{b}}$, Alberto dos Passos Vieira ${ }^{\mathrm{b}}$, \\ Maria Beatrice Gueiros Silva ${ }^{\mathrm{b}}$, Willas Mendonça dos Santos ${ }^{\mathrm{b}}$, Maria Gorete dos Santos Silva ${ }^{\mathrm{b}}$ \\ ${ }^{a}$ Universidade Federal Rural de Pernambuco-UFRPE, Unidade Acadêmica de Garanhuns-UAG, Avenida Bom Pastor, \\ s/n, Boa Vista, Garanhuns-PE. CEP: 55.296-901. E-mail: arsauag@uag.ufrpe.br. \\ ${ }^{\mathrm{b}}$ Universidade Federal Rural de Pernambuco-UFRPE, Unidade Acadêmica de Garanhuns-UAG, Avenida Bom Pastor, \\ s/n, Boa Vista, Garanhuns-PE. CEP: 55.296-901. E-mail: edijailsongoncalves@gmail.com, \\ albertopassosvieira@gmail.com, willia@gmail.com, mgorete@gmail.com.
}

A R T I C L E I N F O

Aceito 15 Mar 2021

Publicado 22 Mar 2021

\begin{abstract}
A B S T R A C T
The Pernambuco semiarid, precipitation, radiation, wind speed and temperature are among the most important meteorological variables for the study of climate change, since their spatial and temporal variability are striking characteristics of the climate. The objective of the study was the correlation structure of meteorological variables to obtain "multivariate climatological indexes" for the municipality of Garanhuns/PE, Brazil, based on the statistical technique of Factorial Analysis (FA) using monthly climatological series from 1963 to 2019 of the meteorological variables, with data from the National Institute of Meteorology (INMET). The statistical procedure was performed using the Multivariate Statistical Analysis technique using the Principal Components method and Hierarchical Conglomerate Cluster Analysis. Using the Factorial Analysis technique that simplified the information contained in the observed variables, related to climatic variability of the region, the multivariate climatic index was obtained. To the values of this index for the municipalities of Garanhuns, the technique of cluster analysis was applied and allowed to identify the formation of five clusters of meteorological variables that more influence the local climate. These groups are characterized by having internal homogeneity and being differentiated from each other. In the same cluster, those with meteorological characteristics more like each other were grouped than with the other variables grouped in the other clusters, where clusters were created that do not violate the principles of homogeneity and separation.
\end{abstract}

Keywords: Weather variables, climate change, southerly wildcat, statistic.

\section{R E S U M O}

No semiárido pernambucano, a precipitação, radiação, velocidade do vento e a temperatura estão entre as variáveis meteorológicas mais importantes para os estudos das mudanças climáticas, uma vez que sua variabilidade espacial e temporal são características marcantes do clima. Objetivou-se estudar a estrutura de correlação de variáveis da área de meteorologia para a obtenção de "Índices climatológicos multivariados" para município de Garanhuns/PE, Brasil, baseado na técnica estatística de Análise Fatorial (AF) utilizando séries climatológicas mensais de 1963 a 2019 das variáveis meteorológicas, com banco de dados proveniente do Instituto Nacional de Meteorologia (INMET). O procedimento estatístico realizado foi à técnica de estatística multivariada de Análise Fatorial utilizando o método das Componentes Principais e Análise de Cluster de Conglomerado Hierárquico. Utilizando a técnica de Análise Fatorial que simplificou a informação contida nas variáveis observadas, relacionadas com variabilidade climática da região, obteve-se o índice do climático multivariado. Aos valores deste índice para os municípios de 
Garanhuns, foi aplicada a técnica de Análise de Agrupamento e permitiu identificar a formação de cinco grupos "cluster" de variáveis meteorológicas que mais influência o clima local. Estes grupos são caracterizados por possuírem homogeneidade interna e por serem diferenciados entre si. Sendo que foram agrupados em um mesmo cluster os com características meteorológicas mais parecidas entre si do que com as demais variáveis agrupadas nos outros clusters, onde foram criados clusters que não violam os princípios da homogeneidade e separação.

Palavras-Chave: Variáveis meteorológicas, mudanças climáticas, agreste meridional, estatística.

\section{Introdução}

O Brasil, por ser um país de grande extensão territorial, possui diferentes regimes de precipitação. De norte a sul, nota-se uma grande diversidade de climas com diferentes características regionais, e devido à ausência de estações meteorológicas em diversas localidades do país, principalmente nos estados da Região do Nordeste Brasileiro (NEB), inúmeros pesquisadores vêm adotando métodos estatísticos, que visam à caracterização climática da região (Bezerra et al., 2008; Freire, et al., 2015; Lacerda, 2015; Camelo et al., 2017).

A porção semiárida da região Nordeste do Brasil é particularmente vulnerável às variações climáticas; a periodicidade das secas compromete, principalmente, a agricultura de subsistência que, na maior parte, se constitui de plantios em sistema de sequeiro (agricultura que não utiliza irrigação) (IPCC, 2014). As constantes variabilidades climáticas relacionadas à posição da zona de convergência intertropical (ZCIT), ao fenômeno El niño - oscilação sul (ENOS) e as oscilações de Temperatura da Superfície do Mar (TSM) são mecanismos inter-relacionados que causam variabilidade na precipitação e no clima do Nordeste Brasileiro - NEB (Molion \& Bernardo, 2002; Gois et al., 2005). Essas variações intensificam as secas severas, juntamente com as ações antrópicas (mudança do uso do solo e emissão de gases efeito estufa), somam à vulnerabilidade da região denominada de áreas sujeitas às secas, as quais são de grandes potencialidades para atividades agropecuárias (Costa et al., 2010).

$\mathrm{O}$ desenvolvimento do semiárido do nordeste do Brasil, principalmente no Agreste Pernambucano, é fortemente dependente da precipitação pluviométrica e, consequentemente, as suas variações provocam prejuízos econômicos e sociais à população do município de Garanhuns/PE que tem como características climáticas marcantes, as irregularidades, tanto espaciais quanto temporais, do seu regime de chuvas (PBMC, 2013; Lacerda, 2015). Segundo Giongo (2011), a história do semiárido passa pelo entendimento do bioma Caatinga e pela necessidade de se trabalhar com medidas mitigatórias e adaptativas, no atual contexto das mudanças climáticas globais.

$\mathrm{O}$ estado de Pernambuco apresenta considerável déficit hídrico e ocupa uma posição de destaque, no contexto das mudanças do clima e seus efeitos, junto com um passivo ambiental considerado. Isto associado à alta vulnerabilidade e aos efeitos das mudanças do clima (Pernambuco, 2006). Além disto, é um dos estados mais vulneráveis do Brasil, quanto aos efeitos das mudanças do clima, sob a intensificação do processo do avanço do mar e da erosão costeira, da ocorrência de eventos extremos de chuva nas mesorregiões do Sertão ao Litoral, e influência de secas recorrentes (Pernambuco, 2006; IPCC, 2014).

No caso do semiárido de Pernambuco, há evidências do aumento das chuvas torrenciais e concentradas em curtos espaços de tempo (Lacerda et al., 2010). Também, espera-se uma maior frequência de dias secos consecutivos e de ondas de calor decorrentes do aumento na frequência de veranicos na região (Marengo et al., 2011).

Entre diversas variáveis analisadas em uma determinada região, os índices climáticos têm como uma das finalidades a caracterização climática de um local considerado; esses índices climáticos de aridez, de umidade e hídrico, e outros representam parte dessa caracterização, obtidos por meio de variáveis meteorológicas, tais como precipitação pluvial, temperatura, umidade, evapotranspiração, pressão velocidade do vento, entre outros (Freitas, 2009). Portanto, o conhecimento detalhado da distribuição espacial e temporal destas variáveis, nas micro e mesorregiões do Estado de Pernambuco, especialmente das principais variáveis físicas influenciadoras das condições climáticas, é de grande importância para a tomada de decisões por parte dos governos e da sociedade civil.

As condições de tempo e clima afetam diretamente diversas atividades humanas. Assim, o planejamento de atividades como a agricultura, a energia e os recursos hídricos levam à necessidade de realizar confiáveis das condições de tempo e clima. Bates et al. (2008) ponderam que as alterações do clima tendem a alterar a 
quantidade e a qualidade da água no semiárido, com consequências significativas na produção de alimentos e no aumento do risco nas práticas agrícolas de sequeiro. Há tendências de crescimento da propagação de doenças de veiculação hídrica, como também de problemas de abastecimento, de operação de infraestrutura hídrica e de práticas de gestão da água, associadas à diminuição dos recursos hídricos no Nordeste do Brasil (Kundzewicz et al., 2007).

A capacidade de realizar previsões climáticas, a partir das variáveis meteorológicas, tem sido o objetivo de muitos estudos, ao longo do tempo, e vários métodos foram e têm sido utilizados na elaboração de previsões climáticas (Bezerra et al., 2008). Estas previsões quando são realizadas com um adequado grau de precisão, proporcionam aos tomadores de decisão e governos, a possibilidade de antecipar e planejar, inteligentemente, as futuras ações dirigidas aos setores de atividades socioeconômicas e à sociedade como um todo.

Têm-se realizado previsões climáticas, tanto com modelos dinâmicos (Coutinho et al., 2006; Kayano et al., 2011; Longo et al., 2006; Freitas et al., 2012; Freire et al., 2015), como com modelos estatísticos (Pezzi et al., 2000; VarejãoSilva, 2000; Kurnaz, 2004; Gneiting \& Raftery, 2005; Bezerra et al., 2008; Camelo et al., 2008, 2017). Os modelos dinâmicos são representações matemáticas dos processos físicos que visam simular a distribuição e evolução de umidade e energia sobre todo o globo. Por outro lado, os modelos estatísticos de previsão do clima estabelecem relações estatisticamente significativas entre variáveis meteorológicas. Em modelos estatísticos de previsão climática, vários podem ser os preditores utilizados: a altitude geopotencial, a radiação, evapotranspiração, a umidade do ar, a temperatura do ar, os ventos na baixa e na alta troposfera e, o mais comum, a chuva da pré-estação no Nordeste.

Nesse contexto estatístico, em diversas áreas como meteorologia, engenharias, psicologia, sociologia, biologia, medicina e economia, depara-se com grande massa de observações de muitas variáveis para cada elemento de uma amostra (ou população) de indivíduos. É de interesse examinar as inter-relações entre as variáveis. Estas inter-relações podem ser avaliadas, ou pelas covariâncias, ou pelos coeficientes de correlação entre as variáveis. Se o número de variáveis é grande, deseja-se estruturar e simplificar os dados de maneira a conservar o máximo de informação expressa pelas variáveis originais (Hair et al., 2010). Uma solução para este problema é encontrar variáveis hipotéticas que sejam combinações lineares das variáveis observadas e mais convenientemente estudadas, por seu menor número (Corrar et al., 2009).

Considerando os muitos problemas da estatística multivariada da análise fatorial e de componentes principais, após determinar as variáveis hipotéticas ou latentes (fatores), que resumiriam as informações das variáveis originais, o passo seguinte é unir os indivíduos formando grupos homogêneos, utilizando suas características, que são mais semelhantes entre si do que com membros de outros grupos. O ramo da estatística que trata desta análise de dados em vários grupos de indivíduos é a Análise de Agrupamento (Silva et al., 2014). O estudo visa criar um procedimento estatístico capaz de determinar as variáveis meteorológicas de maior influência na formação de cada componente principal (fator), especialmente aquelas influenciadoras das mudanças climáticas, por meio da técnica estatística de Análise Fatorial (AF), de Componentes Principais (ACP) e da Análise Agrupamento (AA). Assim, pretende-se indicar os principais fatores e variáveis que comporão um indicador do clima do Município de Garanhuns/PE, Brasil.

Em meteorologia são empregadas algumas técnicas da análise multivariada como a técnica AF, ACP e AA, que têm sido aplicadas em vários estudos de modelagem estatística. Sua grande vantagem está no fato de a mesma possibilitar a busca de padrões de oscilação conjunta entre dois ou mais campos físicos. A partir daí, pode-se encontrar um conjunto de equações prognósticas e construir um modelo preditor. Sendo assim, o presente estudo tem por objetivos: (a) a obtenção de índices de detecção de mudanças climáticas multivariada, considerando variáveis meteorológicas influenciadora de condições climáticas; (b) a hierarquização dos dados de séries climatológica mensais de variáveis meteorológicas, através da formação de grupos homogêneos segundo os índices climatológicos multivariados.

\section{Material e Métodos}

Índices climatológicos

Para a obtenção de índices de detecção de mudanças climáticas por meio da técnica da análise fatorial foram utilizadas séries históricas de 57 anos, no período de janeiro de 1963 a maio de 2019, com dados mensais correspondentes as 13 variáveis meteorológicas influenciadoras das condições climáticas: direção do vento; velocidade do vento média (mps), velocidade do vento máxima média (mps), evaporação piche $(\mathrm{mm})$, insolação total (hs), nebulosidade média 
(décimos); número de dias de precipitação (qtd), precipitação total $(\mathrm{mm})$, pressão atmosférica média (mbar), temperatura do ar máxima média $\left({ }^{\circ} \mathrm{C}\right)$, temperatura do ar compensada média $\left({ }^{\circ} \mathrm{C}\right)$, temperatura do ar mínima média $\left({ }^{\circ} \mathrm{C}\right)$ e umidade relativa média (\%). Os dados utilizados foram provenientes do banco de dados do INMET. A partir de então, este conjunto de dados será chamado de dados do INMET para facilitar a descrição.

\section{Estatística descritiva e normalidade dos dados}

Procedeu-se a análise estatística descritiva dos 741 dados com o objetivo de caracterizar a normalidade dos mesmos. Este procedimento é importante para o conhecimento preliminar da distribuição na área. O programa Sisvar foi utilizado no cálculo das análises univariadas. Antes da modelagem dos dados pela análise de componentes principais, e a análise de agrupamento, foram verificadas as suposições estatísticas da análise da homogeneidade de variâncias, de normalidade e de estacionariedade. Para isto, os dados foram submetidos aos testes de Cochran, Shapiro-Wilk e regressão linear (teste t), respectivamente, ao nível de probabilidade de 5\% (Bussab \& Morettin, 2010). Quando esses requisitos não se verificaram, a fim de corrigir violações das suposições estatísticas, as adequadas transformações matemáticas foram aplicadas aos dados observados observadas, por meio do emprego do logaritmo das variáveis, visando a estabilizar a variabilidade (homogeneizar variâncias) gerada pela presença de valores discrepantes e normalizar as variáveis analisadas (Chen et al., 2003).

A padronização das variáveis, principalmente quando métricas, devem ser observadas na execução da análise. $\mathrm{O}$ conjunto de dados foi padronizado com o objetivo de eliminar possíveis problemas devido a existência de medidas em diferentes escalas e unidades.

\section{Matrizes de Correlação}

Antes de realizar a AF e ACP, faz-se necessário verificar grau de correlação entre as variáveis. A partir da matriz de correlação ou da matriz de variância-covariância, foram realizadas as análises da estrutura de interdependência ente às variáveis. Além da matriz de correlação outras duas medidas foram utilizadas para o exame de intercorrelações entre as variáveis, elaboraram-se os testes de esfericidade de Bartlett e Measure of Sampling Adequacy (MSA) conhecido como teste Kaiser-Meyer-Olkin (KMO), para verificar se as características dos dados são adequadas para proceder a $\mathrm{AF}$ e a $\mathrm{ACP}$, ou seja, indicar o grau de explicação dos dados a partir dos fatores encontrados na AF e na ACP (Maxwell, 2017; Cordeiro et. al., 2017). O teste MSA foi obtida pela aplicação da Equação 1.

$\mathrm{MSA}_{\mathrm{i}}=\sum \mathrm{r}_{\mathrm{ij}}^{2} /\left(\sum \mathrm{r}_{\mathrm{ij}}^{2}+\sum \mathrm{a}_{\mathrm{ij}}^{2}\right)$

onde $\mathrm{r}_{\mathrm{ij}}$ e $\mathrm{a}_{\mathrm{ij}} \mathrm{s}$ sa, respectivamente, o coeficiente de correlação simples e o coeficiente parcial de correlação entre as variáveis i e j, com $\mathrm{i} \neq \mathrm{j}$.

\section{Análise fatorial (AF) para elaboração de índices climatológicos}

A técnica da AF propiciou a identificação de dimensões isoladas da estrutura dos dados, indicando em seguida o grau em que cada variável é elucidada por dimensão ou fator (Hair et al., 2010). A partir da Equação (2) a composição do modelo de análise fatorial foi estimada:

$\mathrm{X}_{\mathrm{i}}=\sum_{\mathrm{j}=1}^{\mathrm{m}} \mathrm{a}_{1 \mathrm{j}} \mathrm{F}_{\mathrm{j}}+\mathrm{U}_{\mathrm{i}}+\mathrm{E}_{\mathrm{i}}$

Eq.(2)

onde $\mathrm{Xi}=\mathrm{a}$ i-ésima variável observável de $\mathrm{i}=1,2$ ,..., p; $\mathrm{a}_{\mathrm{ij}}$ é a carga fatorial (coeficientes dos fatores) da i-ésima variável no j-ésimo fator comum de $\mathrm{j}=1,2, \ldots, \mathrm{m}$ e refletem a importância do j-ésimo fator na composição da i-ésima variável; $F_{j}=$ fatores comuns, $E_{i}=$ fatores específicos, descrevem a variação residual específica da i-ésima variável, resíduo que afeta somente $\mathrm{X}_{\mathrm{i}}$.

A equação (1) pode ser expresso na forma matemática através de uma combinação linear entre as variáveis $\left(\mathrm{X}_{\mathrm{i}}\right)$ e $\mathrm{m}$ fatores comuns $\left(\mathrm{F}_{\mathrm{j}}\right)$ (Hair et al., 2010):

$\mathrm{X}_{1}=\mathrm{a}_{11} \mathrm{~F}_{1}+\mathrm{a}_{12} \mathrm{~F}_{2}+\ldots+\mathrm{a}_{1 \mathrm{~m}} \mathrm{~F}_{\mathrm{m}}+\mathrm{U}_{1}+\mathrm{E}_{1}$

$\mathrm{X}_{2}=\mathrm{a}_{21} \mathrm{~F}_{1}+\mathrm{a}_{22} \mathrm{~F}_{2}+\ldots+\mathrm{a}_{2 \mathrm{~m}} \mathrm{~F}_{\mathrm{m}}+\mathrm{U}_{2}+\mathrm{E}_{2}$

$\mathrm{X}_{3}=\mathrm{a}_{31} \mathrm{~F}_{1}+\mathrm{a}_{32} \mathrm{~F}_{2}+\ldots+\mathrm{a}_{2 \mathrm{~m}} \mathrm{~F}_{\mathrm{m}}+\mathrm{U}_{3}+\mathrm{E}_{3}$

ou seja,

$\mathrm{X}_{\mathrm{i}}=\mathrm{a}_{\mathrm{i} 1} \mathrm{~F}_{1}+\mathrm{a}_{\mathrm{i} 2} \mathrm{~F}_{2}+\ldots+\mathrm{a}_{\mathrm{jm}} \mathrm{F}_{\mathrm{m}}+\mathrm{U}_{\mathrm{i}}+\mathrm{E}_{\mathrm{i}}$

onde $\mathrm{X}_{\mathrm{j}}=\mathrm{i}$-ésima variável; $\mathrm{a}_{\mathrm{j} 1}, \mathrm{a}_{\mathrm{j} 2} \ldots, \mathrm{a}_{\mathrm{jm}}=$ cargas dos fatores para a i-ésima variável, usadas para combinar linearmente os fatores comuns; $F_{1}, F_{2} \ldots$, $\mathrm{F}_{\mathrm{m}}=\mathrm{m}$-ésimo fatores comuns não correlacionados, com "m" menor que "p". Os " $p$ " valores observados das variáveis, são expressos em termos de $\mathrm{p}+\mathrm{m}$ variáveis aleatórias não observáveis $\left(\mathrm{F}_{1}, \mathrm{~F}_{2} \ldots, \mathrm{F}_{\mathrm{m}} ; \mathrm{E}_{1}, \mathrm{E}_{2} \ldots, \mathrm{E}_{\mathrm{p}}\right) ; \mathrm{U}_{\mathrm{i}}=$ fator único e $E_{i}=$ fator o erro de observação, de mensuração ou de especificação do modelo.

A variância comum ou comunalidade $\left(\mathrm{hi}^{2}\right)$ foi utilizada como critério para validação das variáveis no ajuste do modelo fatorial e foi 
determinada pela estimativa da variância de Xi explicada através dos fatores comuns dada pela Equação 4.

$\mathrm{hi}^{2}=\mathrm{a}_{\mathrm{i} 2}^{2}+\mathrm{a}_{12}^{2}+\ldots+\mathrm{a}_{1 \mathrm{~m}}^{2}$

onde $h^{2}{ }^{2}=$ comunalidade; $a_{j 1}, a_{j 2} \ldots, a_{j m}$ são as cargas dos fatores para a i-ésima variável para os "m" fatores.

Através da utilização do programa BioEst v.5.0, foram obtidos os fatores e selecionados os que apresentaram valores maior que 1 . Identificados os fatores, é feita a estimação do escore fatorial, por meio do método semelhante ao da regressão. O escore para cada observação (ano) é resultado da multiplicação do valor (padronizado) das variáveis pelo coeficiente do escore fatorial correspondente, sendo a expressão geral para estimação do j-ésimo fator Fj dada pela Equação 5.

$\mathrm{F}_{\mathrm{j}}=\mathrm{W}_{\mathrm{j} 1} \mathrm{X}_{1}+\mathrm{W}_{\mathrm{j} 2} \mathrm{X}_{2}+\ldots+\mathrm{W}_{\mathrm{jp}} \mathrm{X}_{\mathrm{p}}+\mathrm{U}_{\mathrm{i}}+\mathrm{E}_{\mathrm{i}}$

onde: $\mathrm{W}_{\mathrm{jp}}=$ coeficientes dos escores fatoriais $\mathrm{e}$ "p" é o número de variáveis.

A partir da matriz dos escores fatoriais foi possível construir um índice para hierarquizar as observações da matriz de dados, composta por 741 valores, correspondentes as 13 variáveis meteorológicas e para as 57 observações (anos) no período de 1963 a 2019 do município de Garanhuns/PE. (Monteiro \& Pinheiro, 2004; Melo \& Parré, 2007).

\section{Critérios para a definição do número de fatores}

A opção selecionada para a obtenção dos fatores foi o Método das Componentes Principais. Neste estudo foram utilizados dois critérios para a definição do número de fatores ou componentes principais: critério de Kaiser e o critério da porcentagem da Variância Explicada (Corrar et al., 2009). Pelo critério de Kaiser a seleção do número de fatores foi extraída a partir da matriz de correlação mantidas no sistema apenas as componentes relacionadas aos autovalores $(\lambda \mathrm{i})$ maior e igual a 1, são considerados adequados para extração de fatores. Quanto ao critério da percentagem da variância explicativa, determinouse o número de fatores que explicavam uma porcentagem pré-definida da variabilidade global, segundo Andrade et al. (2018). É comum adotar um valor de explicação de $70 \%$ como mínimo. Porém, este pode mudar de acordo com a área do problema (Ribas \& Vieira, 2011). No caso da correlação dos fatores com as variáveis de produção e qualidade de frutos do melão, consideraram-se as variáveis mais significativas, aquelas com os valores dos coeficientes de correlações superiores a 0,7 , conforme Hair et al. (2010).

\section{Análise de Agrupamento ("Cluster Analysis")}

Obtidos os índices de detecção de mudanças climáticas multivariado para o município de Garanhuns, os valores escores fatoriais de cada fator obtidos através da Análise Fatorial foram analisados pela Análise de Agrupamento. A técnica de análise de Cluster (análise de agrupamento) foi aplicada à matriz dos escores dos fatores com a finalidade agrupar regiões ou locais ou ainda variáveis de acordo com a similaridade de seus perfis; e agrupar variáveis meteorológicas para delinear padrões de variações semelhantes.

O objetivo da aplicação desta técnica multivariada no estudo foi em função de um grande número de objetos ou indivíduos, cada um descrito por um conjunto de medidas, obter um esquema de classificação para agrupar os indivíduos em um número de classes tal que os indivíduos dentro das classes fossem similares, em algum aspecto, e diferentes das outras classes. $\mathrm{O}$ número de grupos e as características de cada grupo devem ser determinados por meio de procedimentos matemáticos. Em suma, esta técnica agrupa elementos de modo a formar grupos homogêneos dentro deles e heterogêneos entre eles.

Para a formação de grupos ou casos mais homogêneos em função das variáveis meteorológicas medidas, não foi possível estabelecer, a priori, o número ideal de grupos a serem formados. Nesse caso, os métodos não hierárquicos são inconvenientes quando comparado com Método Aglomerativo Hierárquico (HCA), pois sua aplicação exigiria grande esforço de cálculo nas sucessivas tentativas para encontrar o número adequado de agrupamentos. Assim, adotou- se o método HCA, que é o mais utilizado na construção de agrupamentos (Meireles \& Oliveira, 2011; Figueiredo Filho et al., 2013).

Por meio do método HCA, as amostras ou variáveis foram agrupadas de acordo com suas similaridades com a distância como a diferença entre os grupos. As medidas de distância, foram utilizadas como medidas de similaridade ou dissimilaridade entre os objetos da matriz de dados. 
Medida de similaridade ou dissimilaridade

Foi utilizada a distância euclidiana como método para medir a dissimilaridade existente para a formação dos grupos de variáveis homogêneas entre as 13 variáveis meteorológicas influenciadoras das condições climáticas, através do programa $\mathrm{R}$. A distância euclidiana $\left(\mathrm{d}_{\mathrm{ij}}\right)$ entre dois indivíduos (i e j) é dada como a raiz quadrada do somatório das diferenças entre os valores de i e j para " $n$ " observações em cada ano, proposta por Ward (1963) (Equação 6).

$\mathrm{d}_{\mathrm{ij}}=\sqrt{\sum_{\mathrm{k}=1}^{\mathrm{n}}\left(\mathrm{X}_{\mathrm{ik}}-\mathrm{X}_{\mathrm{jk}}\right)^{2}}$

onde $\mathrm{d}_{\mathrm{ij}}=$ medida de distância euclidiana do objeto A ao $\mathrm{B} ; \mathrm{j}=$ indexador das variáveis; e $\mathrm{X}_{\mathrm{ik}} \mathrm{e}$ $\mathrm{X}_{\mathrm{jk}}=$ valores das variáveis meteorológicas medidas $\mathrm{X}$ e $\mathrm{Y}$, respectivamente.

Esta distância não possui um valor limite, mas é sempre positiva ou nula. Quanto mais próximo de zero for a distância, maior a similaridade entre os objetos em comparação. A determinação de valores de pequenas dimensões foi utilizada como indicativo da existência de uma forte semelhança entre as variáveis (Boscarioli, 2008).

\section{Método de ligação entre os objetos}

Foi utilizado o método de Ward ou Ward linkage (Ligação simples) para promover a ligação entre os grupos (anos com índices de detecção de mudanças climáticas multivariado homogêneos obtidos com os valores dos escores do modelo fatorial), esse método baseia-se na análise de variância, associando os objetos aos grupos nos quais estes promovem a menor variância intragrupo (Ward, 1963) citado por Figueiredo Filho et al. (2013). O cálculo da variância foi obtido pela Equação (7).

$\mathrm{W}=\sum_{\mathrm{k}=1}^{\mathrm{n}} \mathrm{d}_{\mathrm{ij}}^{2}-\frac{1}{\mathrm{n}}\left(\sum_{\mathrm{k}=1}^{\mathrm{n}} \mathrm{d}_{\mathrm{ij}}\right)^{2}$

De posse dos valores das distâncias euclidianas e das variâncias intergrupo pelo método Ward, foi gerado o dendrograma para representar a árvore ou a história de agrupamento, permitindo a visualização das distâncias entre as séries históricas de 57 anos no período de janeiro de 1963 a maio de 2019 em relação as 13 variáveis meteorológicas influenciadora das condições climáticas envolvidas em cada um dos anos coletados.

O dendrograma corresponde à representação bidimensional do esquema da associação sucessiva dos objetos, atendendo à sua similaridade, até culminar na fusão de todos os grupos num único grupo final. São especialmente úteis na visualização de semelhanças entre objetos ou grupos representados por pontos no espaço com dimensão maior do que três, onde a representação de gráficos convencionais não é possível (Corrar et al., 2009). No eixo das abscissas do dendrograma foram representados os grupos ou regiões (preferencialmente na ordem em que foram agrupados), enquanto, no eixo das ordenadas, foram representadas as dissimilaridades (distâncias) ou a variância entre os grupos. A altura das barras coincide com a distância do agrupamento.

\section{Número de grupos}

Foi utilizado como critério para determinar o número de grupos o exame do dendrograma por meio do critério matemático da inércia para divisão prévia de grupos. A estratégia foi cortar o dendrograma em alguns pontos, observando o número de grupos e o tamanho do intervalo do coeficiente de similaridade/dissimilaridade. Foi escolhido o intervalo de maior tamanho, entre os diversos cortes, ou seja, para obter "k" grupos, basta cortando as k-1 arestas mais altas do dendrograma (Mingoti, 2007; Freitas \& Prata, 2007).

Proposição do método de análise para obtenção do indicador de mudança climática

A verificação do percentual de contribuição de cada variável meteorológica estudada do município de Garanhuns foi feita através dos escores fatoriais, ou seja, dos valores dos fatores para cada uma das 57 observações (anos) e dos valores da variância comum, ou comunalidade $\left(\mathrm{hi}^{2}\right)$, que representa quanto da variância total de cada variável meteorológica $\left(\mathrm{X}_{\mathrm{i}}\right)$ é reproduzida pelos fatores comuns $\left(\mathrm{F}_{\mathrm{j}}\right)$.

Como procedeu-se à análise fatorial pelo método de componentes principais (que faz com que o primeiro fator contenha o maior percentual de explicação da variância total das variáveis da amostra, o segundo fator contenha o segundo maior percentual, e assim por diante), a ponderação pela proporção de explicação da variância total exprimiu a importância relativa de cada fator.

Utilizando-se dos valores da matriz escores fatoriais, foi possível calcular o índice de mudanças climáticas multivariado - IC(Fij) de cada fator, onde cada variável que compõe esse fator foi ponderada por sua carga fatorial por meio da Equação 9. O IC(Fij) é calculado por meio da média ponderada dos escores fatoriais para cada 
observação. A ponderação é realizada a partir da raiz característica de cada fator $\left(\lambda_{\mathrm{jm}}\right)$.

$$
\operatorname{IC}(F i j)=\frac{\sum_{j=1}^{m} \lambda_{j m} . F i j}{\sum_{j=1}^{m} \lambda_{j m}}
$$

onde $\mathrm{IC}(\mathrm{Fij})=$ índice de detecção de mudanças climáticas multivariado (média ponderada dos escores fatoriais) da i-ésima ponto de observação dos " $m$ " fatores extraídos, $0 \leq \mathrm{IC}(\mathrm{Fij}) \leq 1 ; \lambda_{\mathrm{jm}}=\mathrm{j}$ ésima raiz característica dos " $\mathrm{m}$ " fatores extraídos, ou seja, é a proporção da variância explicada por cada fator; $\mathrm{m}=$ número de fatores extraídos na análise; $F i j=$ j-ésimo escore fatorial padronizado da i-ésima ponto de observação e $\Sigma \lambda_{\text {jm }}=$ somatório das raízes características referentes aos "m" fatores extraídos. A participação relativa do fator $\mathrm{j}$ na explicação da variância total captada pelos "m" fatores extraídos é indicada por: $\lambda_{\text {jm }} /$ $\Sigma \lambda_{\text {jm. }}$

O IC(Fij) é apresentado em forma percentual variando de 0 (quanto a condições climática da região está no limite inferior da escala, ou seja, apresentam uma baixa previsibilidade climática) a 100 (alta previsibilidade climática, ou seja, é um indicador de alta destreza por parte do modelo preditor). Para isto, é necessário fazer a padronização do escore fatorial, para se obter valores positivos dos escores originais sendo possível hierarquizar os municípios, dado que os valores do IC(Fij) estão entre zero e um. A fórmula para a padronização dos escores:

$$
\text { Fij }=\frac{\text { Fj }- \text { Fmín }}{\text { Fmáx }- \text { Fmín }}
$$

onde: $\mathrm{Fj}=$ escore fatorial original; Fmín $=$ valor mínimo observado para os escores fatoriais; Fméx = valor máximo observado para os escores fatoriais.

Assim, a escala hierárquica de classificação das observações: IC(Fij) igual ou maior que 0,70 são considerados altos, se referindo ao fator ou variável latente com alto grau de mudanças climáticas; valores entre 0,40 e 0,69 considera-se intermediários e; valores abaixo de 0,40 são os fatores que apresentam baixo IC(Fij).

\section{Resultados e Discussão}

Análise estatística univariada

As variáveis utilizadas para esse estudo definiram, de um modo geral, o comportamento das condições climáticas; a estatística descritiva foi realizada nas variáveis para dar uma noção geral do comportamento das variáveis e também foi necessário entender a distribuição dessas variáveis para determinar medidas importantes para a execução da Análise Fatorial. A Tabela 1 apresenta as estatísticas descritivas das 13 variáveis analisadas.

Tabela 1. Parâmetros estatísticos relativos das variáveis meteorológicas referentes à série histórica de 57 anos, no período de janeiro de 1963 a maio de 2019, do município de Garanhuns/PE, Brasil. Fonte: Andrade et al. (2020).

\begin{tabular}{lcccccccc}
\hline Variáveis & Vmín & Vmáx & Média & Md & Ak & Ck & DP & CV \\
\hline DV & 11,00 & 17,00 & 14,00 & 14,00 & $-0,15$ & $-1,17$ & 2,10 & 15,02 \\
VVM & 1,47 & 4,80 & 3,49 & 3,37 & $-0,32$ & 0,30 & 0,66 & 19,02 \\
VVMM & 4,37 & 9,86 & 6,97 & 6,83 & 0,29 & 0,90 & 1,06 & 15,17 \\
EVp & 33,14 & 180,42 & 98,10 & 86,61 & 0,65 & $-0,33$ & 37,79 & 38,52 \\
INS & 35,87 & 232,71 & 154,04 & 165,13 & $-0,51$ & $-0,74$ & 50,89 & 33,03 \\
NEBM & 3,14 & 11,82 & 6,98 & 6,98 & $-0,03$ & $-0,23$ & 1,94 & 27,76 \\
NDP & 0,67 & 28,44 & 10,17 & 9,56 & 0,46 & $-0,42$ & 6,80 & 66,91 \\
PT & 0,57 & 85,20 & 29,82 & 27,36 & 0,43 & $-0,42$ & 20,56 & 68,94 \\
PNMM & 914,64 & 925,54 & 921,37 & 922,04 & $-1,60$ & 2,89 & 2,19 & 0,24 \\
TMaM & 20,01 & 32,97 & 26,22 & 25,83 & 0,61 & 0,63 & 2,61 & 9,93 \\
TCM & 15,41 & 30,02 & 22,20 & 21,65 & 1,05 & 1,42 & 2,88 & 12,96 \\
TMiM & 12,45 & 27,00 & 19,24 & 18,51 & 0,97 & 1,45 & 2,77 & 14,39 \\
UR & 55,33 & 96,09 & 78,59 & 80,70 & $-0,80$ & 0,12 & 9,20 & 11,70 \\
\hline
\end{tabular}

Vmín = valor mínimo; Vmáx = valor máximo; $\mathrm{Md}=$ mediana; $\mathrm{Ak}=$ coeficiente de assimetria; $\mathrm{Ck}=$ curtose; $\mathrm{DP}=$ desvio-padrão; CV = Coeficiente de variação (\%); DV = Direção do vento (gruas); VVM = Velocidade do vento média (mps); VVMM = Velocidade do vento máxima média (mps); EVp = Evaporação (mm); INS = Insolação total (horas); NEBM = Nebulosidade média (décimos); NDP = Número de dias com precipitação (qtd); PT = precipitação total $(\mathrm{mm}) ; \mathrm{PNMM}=$ Pressão atmosférica ao nível do mar média (mbar); TMaM = 
Temperatura do ar máxima média $\left({ }^{\circ} \mathrm{C}\right) ; \mathrm{TCM}=$ Temperatura do ar compensada média $\left({ }^{\circ} \mathrm{C}\right) ; \mathrm{TMiM}=$ Temperatura do ar mínima média $\left({ }^{\circ} \mathrm{C}\right)$ e UR $=$ Umidade relativa média $(\%)$.

As medidas de tendência central (média e mediana) foram semelhantes para mais de 69,23 $\%$ das variáveis medidas, indicando uma distribuição normal e, consequentemente, os dados apresentaram reduzido afastamento em relação a um valor central. Os valores de média e mediana, para grande parte dos atributos, próximos, indicando distribuições simétricas, o que pode ser confirmado pelos valores de assimetria próximos de zero, com exceção das variáveis PNMM, TCM, TMiM e UR, que apresentaram distribuições assimétricas.

Pode-se concluir dois pontos importantes do resultado da estatística descritiva. O primeiro é que as variáveis estão em unidade de medidas muito diferentes e desproporcionais, impossibilitando o uso da matriz de covariância para a Análise Fatorial; é notório entre, por exemplo, a mediana das variáveis NEBM e PNMM, com valores de 6,98 (decimais) e 922,04 (mbar) respectivamente. $\mathrm{O}$ segundo fato importante do resultado da estatística descritiva é a assimetria em as variáveis dos nossos dados, que é um indício de utilização do método dos componentes principais na extração dos fatores, por não exigir uma distribuição nos dados (Barroso, 2003).

O teste Shapiro-Wilk obteve-se um pvalor menor $0,001 \%$, esse valor indica que se rejeita a hipótese nula de que não há normalidade nos dados no nível de $0,05 \%$, podendo ser utilizado com qualquer método de extração fatorial. Confirmando a utilização do método de componentes principais na aplicação da análise fatorial. Segundo Fava \& Velicer (1992) normalidade multivariada é uma suposição rígida, necessária somente para a execução de certos tipos de métodos extração de fatores, como, por exemplo, o método de máxima verossimilhança. $\mathrm{E}$ ressaltar que a normalidade multivariada é uma suposição razoável somente nos casos em que cada variável apresenta normalidade univariada.

Os maiores coeficientes de variação (Tabela 2) foram observados para as variáveis NDP e PT, enquanto os elementos PNMM e VVM apresentaram as menores variabilidades para área com atividade agrícola. A variabilidade pode ser útil para identificar padrões na distribuição da concentração destes elementos. Uma maneira de detectar similaridades e diferenças entre as variáveis meteorológicas em uma evolução espacial simultânea é estudando o coeficiente de correlação. Como analisado anteriormente as variáveis são medidas em magnitudes diferentes então a Análise Fatorial será aplicada à matriz de correlações que se encontra na Tabela 2. Quanto mais próximo de 1 estiverem os valores dos coeficientes de correlação mais forte são as correlações entre as variáveis.

Tabela 2. Matriz de correlações das variáveis meteorológicas referentes à série histórica de 57 anos, no período de janeiro de 1963 a maio de 2019, do município de Garanhuns/PE, Brasil. Fonte: Andrade et al. (2020).

\begin{tabular}{lccccccccccccc}
\hline Variáveis & DV & VVM & VVMM & EVp & INS & NEBM & NDP & PT & PNMM TMaM TCM TMiM & UR \\
\hline DV & 1 & $-0,121$ & $-0,159$ & $-0,366$ & $-0,048$ & 0,231 & 0,281 & 0,283 & 0,165 & $-0,092$ & $-0,121$ & $-0,105$ & 0,183 \\
VVM & $-0,121$ & 1 & 0,806 & 0,344 & 0,516 & $-0,613$ & $-0,137$ & $-0,132$ & 0,314 & $-0,57$ & $-0,616$ & $-0,612$ & 0,534 \\
VVMM & $-0,159$ & 0,806 & 1 & 0,186 & 0,257 & $-0,434$ & $-0,198$ & $-0,202$ & 0,195 & $-0,194$ & $-0,218$ & $-0,198$ & 0,198 \\
EVp & $-0,366$ & 0,344 & 0,186 & 1 & 0,638 & $-0,615$ & $-0,689$ & $-0,693$ & $-0,001$ & $-0,16$ & $-0,227$ & $-0,213$ & 0,105 \\
INS & $-0,048$ & 0,516 & 0,257 & 0,638 & 1 & $-0,806$ & $-0,1$ & $-0,098$ & $-0,118$ & $-0,561$ & $-0,566$ & $-0,612$ & 0,445 \\
NEBM & 0,231 & $-0,613$ & $-0,434$ & $-0,615$ & $-0,806$ & 1 & 0,255 & 0,255 & $-0,063$ & 0,481 & 0,48 & 0,493 & $-0,42$ \\
NDP & 0,281 & $-0,137$ & $-0,198$ & $-0,689$ & $-0,1$ & 0,255 & 1 & 0,999 & $-0,128$ & $-0,222$ & $-0,13$ & $-0,194$ & 0,247 \\
PT & 0,283 & $-0,132$ & $-0,202$ & $-0,693$ & $-0,098$ & 0,255 & 0,999 & 1 & $-0,126$ & $-0,234$ & $-0,14$ & $-0,205$ & 0,253 \\
PNMM & 0,165 & 0,314 & 0,195 & $-0,001$ & $-0,118$ & $-0,063$ & $-0,128$ & $-0,126$ & 1 & $-0,425$ & $-0,391$ & $-0,278$ & 0,467 \\
TMaM & $-0,092$ & $-0,57$ & $-0,194$ & $-0,16$ & $-0,561$ & 0,481 & $-0,222$ & $-0,234$ & $-0,425$ & 1 & 0,94 & 0,923 & $-0,882$ \\
TCM & $-0,121$ & $-0,616$ & $-0,218$ & $-0,227$ & $-0,566$ & 0,48 & $-0,13$ & $-0,14$ & $-0,391$ & 0,94 & 1 & 0,97 & $-0,883$ \\
TMiM & $-0,105$ & $-0,612$ & $-0,198$ & $-0,213$ & $-0,612$ & 0,493 & $-0,194$ & $-0,205$ & $-0,278$ & 0,923 & 0,97 & 1 & $-0,816$ \\
UR & 0,183 & 0,534 & 0,198 & 0,105 & 0,445 & $-0,42$ & 0,247 & 0,253 & 0,467 & $-0,882$ & $-0,883$ & $-0,816$ & 1 \\
\hline
\end{tabular}

DV = Direção do vento (gruas); VVM = Velocidade do vento média (mps); VVMM = Velocidade do vento máxima média (mps); EVp = Evaporação $(\mathrm{mm}) ; \mathrm{INS}=$ Insolação total (horas); NEBM = Nebulosidade 
média (décimos); NDP = Número de dias com precipitação $($ qtd); $\mathrm{PT}=$ precipitação total $(\mathrm{mm}) ; \mathrm{PNMM}=$ Pressão atmosférica ao nível do mar média (mbar); TMaM = Temperatura do ar máxima média $\left({ }^{\circ} \mathrm{C}\right) ; \mathrm{TCM}=$ Temperatura do ar compensada média $\left({ }^{\circ} \mathrm{C}\right) ; \mathrm{TMiM}=$ Temperatura do ar mínima média $\left({ }^{\circ} \mathrm{C}\right)$ e UR $=$ Umidade relativa média $(\%)$.

Analisando a de matriz de correlação, consideraram-se como "boa" correlação aqueles coeficientes maiores ou iguais a 0,31 , em valores absolutos (embora, em nível de $\alpha=0,01$, foram estatisticamente significantes todos os coeficientes maiores do que $| \pm 0,168|$ ) (Guedes et al., 2010). $\mathrm{Na}$ Tabela 2 utilizou-se a seguinte convenção: Os coeficientes de correlação maiores ou iguais \pm 0,31 aparecerão na matriz cor cinza caso contrário aparecerá sem textura de cor. A distribuição dos bons coeficientes ilustra quão mais indicadoras ou diagnósticas são certas variáveis em relação às outras dentro de universo total de dados.

Segundo Fávero et al. (2009), a existência de valores dos coeficientes de correlações elevados entre as duas ou mais variáveis analisadas denota a presença de multicolinearidade. Os resultados da Tabela 2 revelaram que não existem problemas de elevadas correlações entre duas variáveis, o que indicou ausência de multicolinearidade. A maior correlação positiva ocorreu entre a composição de temperatura do ar mínima média (TMiM) e as temperatura do ar máxima média (TMaM) e temperatura do ar compensada média (TCM).

\section{Validação do instrumento $e$ análise de confiabilidade}

$\mathrm{O}$ resultado estatístico de KMO foi de 0,61, que segundo Barroso (2003), o índice indica que a solução do modelo fatorial foi possível de ser aplicada às variáveis meteorológicas, e indicando que os componentes principais encontradas conseguiram descrever satisfatoriamente as variações dos dados originais.

$\mathrm{O}$ teste de $\mathrm{KMO}$ apresentou um alto poder de explicação dos dados $(0,747)$, o índice indicou que os dados foram adequados para a realização do modelo fatorial. $O$ teste de esfericidade de Bartlett apresentou valor de 1024,47, com nível de significância alto $(\mathrm{p} \leq 1 \%$ ), e grau de liberdade 66 , que permitiu concluir que a redução do espaço dimensional foi adequada. Segundo Corrar et al. (2009) e Hair et al. (2010), recomenda-se que o valor de Sig (Teste de significância) do teste de Bartlett não ultrapasse 0,05 , para que seja possível a aplicação da AF. O nível de significância do estudo foi nulo, indicando que é possível a aplicação da AF nas variáveis analisadas. Portando, o teste KMO e o de Bartlett mostraram que existe correlação entre as variáveis meteorológicas, e que ambos os resultados dos testes discutidos atenderam os pressupostos da utilização da AF e ACP aos dados.

Outra medida que foi utilizada para quantificar o grau de intercorrelações entre as variáveis foi dada pela estatística da MSA (medida de adequação da amostra), que parte do princípio da matriz anti-imagem, ou seja, as correlações parciais entre as variáveis e, que variam entre 0 a 1 , sendo que, quanto mais próximo de 1 melhor, é a previsão sem erro de cada variável pelas outras. Foi obtido o índice de MSA para cada variável (Tabela 3) e concluiu-se que foi aceitável prosseguir com a análise fatorial, pois todas as variáveis apresentaram um valor maior que 0,5 .

Pela aplicação do teste KMO, quando aplicadas as 13 variáveis analisadas, foram obtidos um resultado insuficiente $(0,501)$ e um valor de comunalidade $(0,386)$ para a variável direção do vento (DV), indicando a necessidade de eliminação "extração" desta variável para que fosse possível a aplicação da análise fatorial.

Tabela 3. Estatística das medidas de adequação de amostragem (MSA) das variáveis meteorológicas referentes à série histórica de 57 anos, no período de janeiro de 1963 a maio de 2019, do município de Garanhuns/PE, Brasil. Fonte: Andrade et al. (2020).

\begin{tabular}{lc}
\hline Variáveis meteorológicas & Valor do MAS \\
\hline VVM & 0,732 \\
VVMM & 0,539 \\
EVp & 0,788 \\
INS & 0,773 \\
NEBM & 0,869 \\
NDP & 0,593 \\
PT & 0,586 \\
PNMM & 0,632 \\
TMaM & 0,883 \\
TCM & 0,776 \\
TMiM & 0,759 \\
UR & 0,826 \\
\hline VVM
\end{tabular}

VVM = Velocidade do vento média (mps); VVMM = Velocidade do vento máxima média $(\mathrm{mps}) ; \mathrm{EVp}=$ Evaporação $(\mathrm{mm}) ; \mathrm{INS}=$ Insolação total (horas); NEBM = Nebulosidade média (décimos); $\mathrm{NDP}=$ Número de dias com precipitação (qtd); $\mathrm{PT}=$ precipitação total $(\mathrm{mm})$; PNMM = Pressão atmosférica ao nível do mar média (mbar); TMaM = Temperatura do ar 
máxima média $\left({ }^{\circ} \mathrm{C}\right) ;$ TCM $=$ Temperatura do ar compensada média $\left({ }^{\circ} \mathrm{C}\right) ; \mathrm{TMiM}=$ Temperatura do ar mínima média $\left({ }^{\circ} \mathrm{C}\right)$ e UR $=$ Umidade relativa média $(\%)$.

\section{Análises de fatores $(A F)$ e de componentes principais $(A C P)$}

Para a extração dos fatores foi utilizado o método de componentes principais (ACP), visto que não exige uma distribuição nos dados. A Tabela 4 mostra os fatores ou dimensões latentes na AF através dos critérios de Kaiser (raízes características ou autovalores) e das proporções da variância total explicada e acumuladas, obtidos pelo método da ACP, segundo variáveis padronizadas para os dados após rotação.

Tabela 4. Autovalores, autovetores, cargas fatoriais, comunalidades e variância explicada na extração de fatores ou componentes principais pela análise fatorial (AF) das variáveis meteorológicas referentes à série histórica de 57 anos, no período de janeiro de 1963 a maio de 2019, do município de Garanhuns/PE, Brasil. Fonte: Andrade et al. (2020).

\begin{tabular}{lcccc}
\hline \multirow{2}{*}{ Estatística } & \multicolumn{4}{c}{ Fatores extraídos rotacionados com seus autovalores } \\
\hline Autovalores & F1 & F2 & F3 & F4 \\
\% de variância & 4,706 & 2,836 & 1,966 & 1,509 \\
\% acumulativa & 39,21 & 23,63 & 16,38 & 12,57 \\
\hline Variáveis & 39,21 & 62,84 & 79,22 & 91,79 \\
\hline VVM & $\mathbf{F 1}$ & $\mathbf{2}$ & Autovetores & $\mathbf{F 4}$ \\
VVMM & 0,025 & 0,056 & 0,454 & 0,024 \\
EVp & 0,178 & 0,114 & 0,669 & 0,046 \\
INS & $-0,065$ & $-0,298$ & $-0,134$ & $-0,181$ \\
NEBM & $-0,09$ & $-0,04$ & $-0,031$ & $-0,419$ \\
NDP & 0,038 & 0,065 & $-0,122$ & 0,279 \\
PT & $-0,019$ & 0,369 & 0,046 & $-0,14$ \\
PNMM & $-0,022$ & 0,369 & 0,042 & $-0,137$ \\
TMaM & $-0,15$ & $-0,142$ & 0,006 & 0,6 \\
TCM & 0,235 & $-0,009$ & 0,085 & $-0,055$ \\
TMiM & 0,236 & 0,02 & 0,088 & $-0,057$ \\
UR & 0,218 & $-0,017$ & 0,076 & 0,035 \\
\hline
\end{tabular}

$\mathrm{F}$ = fatores ou componentes principais; VVM = Velocidade do vento média (mps); VVMM = Velocidade do vento máxima média (mps); EVp = Evaporação (mm); INS = Insolação total (horas); NEBM = Nebulosidade média (décimos); NDP = Número de dias com precipitação (qtd); PT = precipitação total $(\mathrm{mm}) ; \mathrm{PNMM}=$ Pressão atmosférica ao nível do mar média (mbar); TMaM = Temperatura do ar máxima média $\left({ }^{\circ} \mathrm{C}\right) ; \mathrm{TCM}=$ Temperatura do ar compensada média $\left({ }^{\circ} \mathrm{C}\right) ; \mathrm{TMiM}=$ Temperatura do ar mínima média $\left({ }^{\circ} \mathrm{C}\right)$ e UR $=$ Umidade relativa média $(\%)$.

Segundo Ayres (2012), os autovalores são as variâncias dos componentes principais e representam o poder explicativo do componente em relação à variância das variáveis originais (observadas), ou seja, representam o comprimento dos eixos dos componentes principais de um conjunto de dados e são medidos em unidades de variância.

Os autovetores são os coeficientes das variáveis $\mathrm{X}_{\mathrm{i}}$ padronizados usados para calcular escores dos componentes principais. Os autovetores representam o módulo unitário associado a cada autovalor e as direções dos eixos dos componentes principais (Manly, 2008; Ayres, 2012). Os coeficientes de autovetores constantes da Tabela 4 foram utilizados para ponderar as variáveis (pesos) que são utilizados nos cálculos dos índices climatológicos multivariados.

A Tabela 4 apresenta orientações (autovalores, percentual da variância total explicada e acumulada) para identificações dos fatores a serem extraídos, com base na AF. Pelo critério de Kaiser, todos os autovalores maiores que o valor 1 devem ser incluídos na $\mathrm{AF}$, neste caso seriam quatro fatores extraídos. $\mathrm{O}$ outro critério, o método da variância explicada, seguindo o valor de pelo menos $70 \%$ da variância acumulada explicada, apenas três fatores seriam extraídos. Entretanto, esse critério é muito subjetivo, pois o valor sugerido de $70 \%$ muda conforme diferentes literaturas. $\mathrm{O}$ resultado desta variância foi satisfatório, segundo Hair et al. 
(2010), pois obter uma variância acumulada de $70 \%$ é satisfatório para a extração de fatores.

Entre os dois métodos, a melhor escolha de extração estaria entre três ou quatro fatores para a análise, neste sentido foi utilizado o gráfico Scree Plot (Figura 1). A Figura 1 identifica o "cotovelo" no $4^{\text {a. }}$ fator. A partir dos três critérios foi decidida a extração de quatro fatores, pois quatro é um valor em comum em todos os critérios, como o critério de Kaiser é baseado em critérios matemáticos, o que o torna mais confiável.

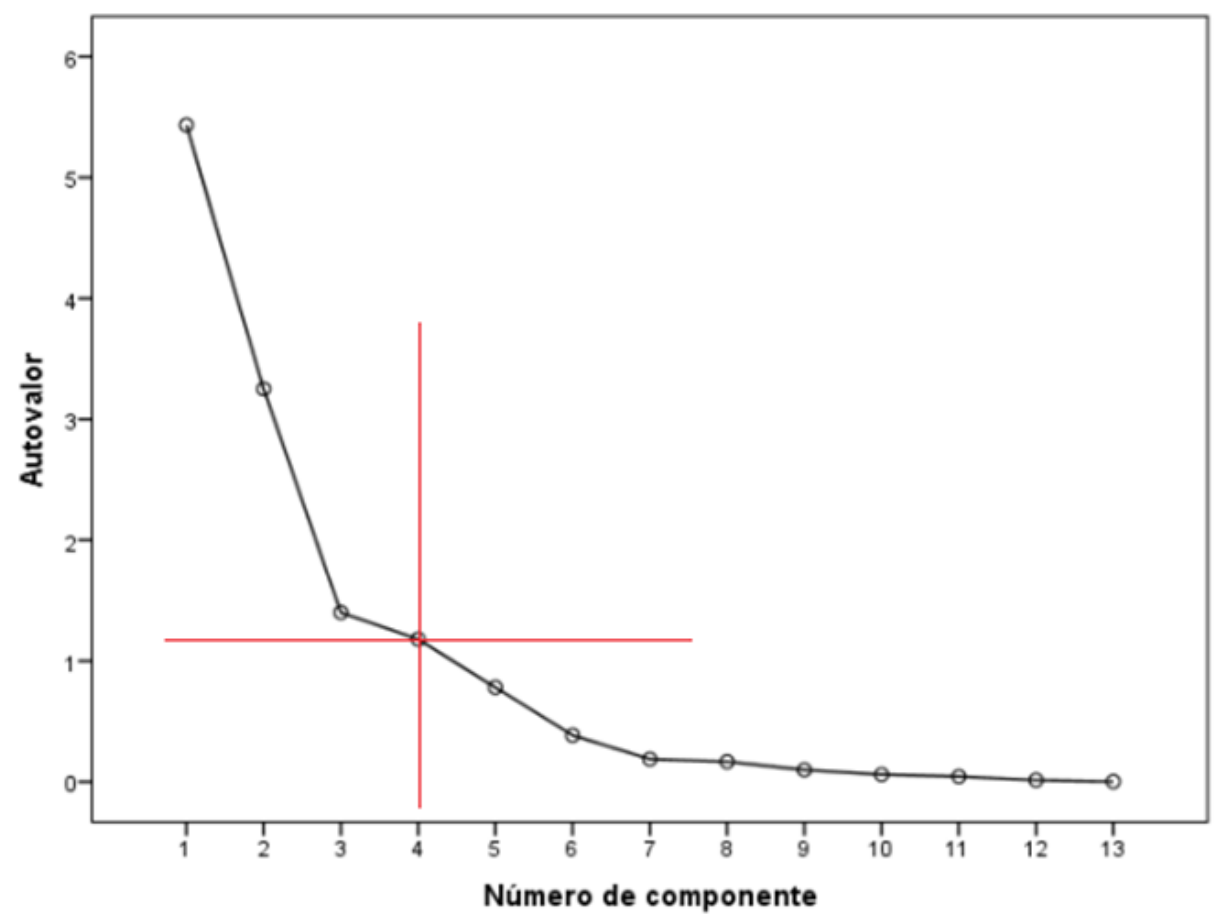

Figura 1. Método de Scree Plot como critérios de extração de fatores ou componente. Fonte: Andrade et al. (2020).

Verificou-se que o primeiro e o segundo fatores se relacionam fortemente com algumas variáveis, os outros fatores contêm relações moderadas com as outras variáveis, dificultando a interpretação e a importância de cada fator (Tabela 4). Nesta situação, foi utilizada a rotação dos fatores Varimax, pois melhora a interpretação da relação entre os fatores e acentua a relação das variáveis com apenas um fator.

Após a extração e rotação dos fatores (Tabela 4), o modelo conseguiu explicar, aproximadamente, $\quad 91,79 \%$ da variância acumulada total das variáveis originais e os autovalores com valores superiores a 1 . De acordo com o resultado da aplicação da análise fatorial com a extração dos fatores, utilizando o método das componentes principais, dentre os 13 fatores ou componentes principais gerados, optou-se por utilizar quatro fatores. Estes quatro fatores foram rotacionado por meio da rotação Varimax com Kaiser normalizada, para facilitar a interpretação da contribuição das variáveis (pesos) em cada fator $\left(\mathrm{F}_{\mathrm{j}}\right)$.

A Tabela 5 apresenta os pesos "cargas fatoriais" dos fatores rotacionados maiores que 0,400 e menores que $-0,400$, para facilitar a identificação das variáveis com maior importância na combinação linear de cada fator, bem como as comunalidades representam a proporção da variância comum dentro da variável (Field, 2009). Assim, observa-se, na Tabela 5, que 0,947 $(94,70 \%)$ da variância associada à variável temperatura do ar compensada média (TCM) é uma variância comum ou compartilhada nos quatro primeiros fatores. Isto significa o poder de explicação da variável em termos do coeficiente de determinação $\left(\mathrm{R}^{2}\right)$, o que indica a importância da variável. A variável precipitação total (PT) com a comunalidade de $0,972(97,20 \%)$ é a mais importante dos quatro primeiros fatores, por compartilhar mais de sua variância.

Tabela 5. Cargas fatoriais ou coeficientes de correlação entre as variáveis meteorológicas e as componentes principais (CP), após rotação Varimax para os dados da série histórica de 57 anos, no período de janeiro de 1963 a maio de 2019, do município de Garanhuns/PE, Brasil. Fonte: Andrade et al. (2020). 


\begin{tabular}{|c|c|c|c|c|c|}
\hline & F1 & F2 & F3 & F4 & \\
\hline TCM & 0,961 & -------- & ------- & ------- & 0,947 \\
\hline TMaM & 0,960 & ------- & -------- & ------- & 0,946 \\
\hline TMiM & 0,935 & -------- & ------- & ------- & 0,916 \\
\hline UR & $-0,913$ & ------- & -------- & ------- & 0,879 \\
\hline PT & -------- & 0,959 & ------- & ------- & 0,972 \\
\hline NDP & ------- & 0,958 & ------- & ------- & 0,967 \\
\hline $\mathrm{EVp}$ & ------- & $-0,830$ & ------- & ------- & 0,901 \\
\hline VVMM & ------- & ------- & 0,975 & ------- & 0,966 \\
\hline VVM & $-0,499$ & ------- & 0,813 & ------- & 0,934 \\
\hline PNMM & $-0,452$ & ------- & ------- & 0,777 & 0,868 \\
\hline INS & $-0,581$ & ------- & ------- & $-0,683$ & 0,918 \\
\hline NEBM & 0,486 & ------- & $-0,414$ & 0,497 & 0,801 \\
\hline
\end{tabular}

$\mathrm{F}=$ fatores ou componentes principais; $\mathrm{TCM}=$ Temperatura do ar compensada média $\left({ }^{\circ} \mathrm{C}\right) ; \mathrm{TMaM}=$ Temperatura do ar máxima média $\left({ }^{\circ} \mathrm{C}\right)$; TMiM $=$ Temperatura do ar mínima média $\left({ }^{\circ} \mathrm{C}\right)$; UR = Umidade relativa média (\%); PT = precipitação total $(\mathrm{mm}) ; \mathrm{NDP}=$ Número de dias com precipitação (qtd); EVp = Evaporação $(\mathrm{mm}) ; \mathrm{VVMM}=$ Velocidade do vento máxima média (mps); VVM = Velocidade do vento média (mps); PNMM = Pressão atmosférica ao nível do mar média (mbar); INS = Insolação total (horas); $\mathrm{NEBM}=$ Nebulosidade média (décimos) e $\mathrm{hi}^{2}=$ comunalidade .

A Tabela 5 mostra que não houve fatores correlacionados aos demais fatores (F5 a F13), visto que os coeficientes "cargas fatoriais" determinados a essas componentes mostram valores menores $(\leq 0,40)$ aos apresentados em relação às F1, F2, F3 e F4. Algo bastante importante a ser considerado nas cargas fatoriais destacadas, conforme a Tabela 5, é que algumas delas apresentaram sinal negativo, possivelmente de ser considerado que algumas das variáveis, conforme o fator de maior relevância, têm relação inversa na formulação das mudanças do clima regional "Índices climatológicos".

\section{Construção dos índices climatológicos multivariados}

A base para a obtenção dos índices climatológicos multivariados é a tabela das cargas fatoriais após a rotação (Tabela 5), na obtenção dos escores fatoriais. Os escores fatoriais de cada fator possuem distribuição normal, com média zero e variância unitária e, desse modo, podem ser utilizados para indicar a posição relativa de cada observação, relativamente ao conceito expresso pelo fator. Portanto, pode-se interpretar que os escores com valores próximos de zero indicam nível de influência na mudança climática média e, quanto maior em relação a zero for o fator, mais influência na mudança climática terá a variável estudada, no que se refere ao significado do fator em consideração. Assim, a partir da matriz dos escores fatoriais, é possível construir um índice para hierarquizar as observações (Monteiro \& Pinheiro, 2004). A partir da matriz fatorial das cargas fatoriais com rotação Varimax, as variáveis meteorológicas estão correlacionas com os fatores das seguintes formas: i) Fator 1 (F1): variáveis originais (TCM, TMaM, TMiM e UR) com as mais altas correlações "cargas fatoriais com a Fl

O primeiro fator (F1) explica $39,21 \%$ da variância total dos dados. Os maiores pesos positivos, ou associação a esse fator, são: TCM, TMaM, TMiM e o maior peso negativo é observado apenas na variável umidade relativa do ar (UR). Isto sugere que esse fator representa as propriedades das varáveis relacionadas com aspectos da temperatura e do teor de água na atmosfera, que representa a parte comum mais importante das variáveis meteorológicas analisadas como influenciadora das condições climáticas para o município de Garanhuns/PE, Brasil, e que pode ser denominada "Índices das distribuições espacial e temporal da temperatura do ar e do teor de água na atmosfera". Portanto, este fator pode ser interpretado como responsável pela contribuição da caracterização das variações de temperatura e umidade do ar. As maiores cargas fatoriais positivas nesta componente são os atributos TCM $(0,961)$ e TMaM $(0,960)$. As temperaturas certamente são um dos elementos meteorológicos mais importantes e decisivos sobre os níveis de intensidade da umidade do ar, que afeta diretamente as mudanças climáticas globais e locais. A umidade relativa do ar depende, diretamente, porém inversamente, da quantidade de vapor contido em parcela de ar e da temperatura do ar. Conforme aumenta a umidade absoluta no interior da parcela de ar, a UR aumenta e, por outro lado, conforme aumenta a temperatura do ar, a UR diminui. Em outras palavras, a umidade relativa do ar, medida em porcentagem, aumenta com a redução da 
temperatura, pois as temperaturas mais altas tendem a tornar o ar mais seco e com menos umidade.

Conforme o quinto relatório do Painel Intergovernamental sobre Mudanças do Clima (IPCC, 2014), o aumento nas temperaturas médias, juntamente com a nova composição química da atmosfera, desencadeou alterações significativas no sistema climático planetário, afetando o padrão de chuvas, com impactos no ciclo hidrológico, produzindo enchentes intensas, secas severas e frequentes ondas de frio e calor, com consequências na segurança alimentar, na saúde e na segurança hídrica. A região Nordeste do Brasil, em geral, e o estado de Pernambuco, em especial, estão vulneráveis aos processos de desertificação, à ocorrência de eventos extremos do clima, tais como secas e enchentes e ao avanço do mar, decorrentes do aumento de temperatura.

ii) Fator 2 (F2): variáveis originais (PT, NDP e $E V p)$ com as mais altas correlações "cargas fatoriais com a F2

$\mathrm{O}$ segundo fator (F2) explica $23,63 \%$ da variância total dos dados. Os maiores pesos positivos para esse fator são PT e NDP, e o maior peso negativo é observado apenas da variável $E V p$, sugerindo que essa componente principal representa a parte comum mais importante das variáveis analisadas na obtenção de índices de detecção de mudanças climáticas multivariados para o município de Garanhuns/PE, Brasil, e pode ser denominada "Índice da disponibilidade hídrica". Este fator pode ser interpretado como responsável pela contribuição da caracterização das variações de temperatura e umidade do ar. As maiores cargas fatoriais positivas nesta componente são as varáveis PT $(0,959)$ e NDP $(0,958)$. A carga fatorial da variável evaporação ser negativa $(-0,830)$ é pelo fato que a precipitação total tende a acompanhar o número de precipitação, enquanto a evaporação apresenta tendência inversa à de ambos.

No estado de Pernambuco, a precipitação está entre as variáveis meteorológicas mais importantes para os estudos das mudanças climáticas, uma vez que sua variabilidade espacial e temporal são características marcantes do clima local. Valores de precipitação tendem a acompanhar o número de precipitação, enquanto a evaporação apresenta tendência inversa à de ambos. Segundo Lacerda et al. (2015a), boa parte dos meses do ano, para a região do semiárido do Estado de Pernambuco, apresentaram índices de evaporação bem maiores que os índices de precipitação, demonstrando que ocorre um déficit hídrico na cidade de Garanhuns, pois quanto menor a capacidade de água disponível, maior a deficiência hídrica da região.

De acordo com Marengo et al. (2011), o cenário climático brasileiro acompanha a mesma tendência de aquecimento global, em que as mudanças mais significativas são o aumento de temperatura, modificações nos padrões de chuvas e alterações na distribuição de extremos climáticos, tais como secas, enchentes e inundações. Sob essa ótica, as análises das variações de chuva e de temperatura, bem como seus impactos no balanço hídrico, servirão como base para a detecção dos efeitos do aquecimento global, mudanças climáticas locais e consequentes impactos. Um impacto inquestionável é o aumento da evapotranspiração com o aumento da temperatura. Esse aumento tende a ser especialmente danoso às atividades agrícolas, pois reduziria a água disponível para as plantas, no solo.

Na região, as consequências sobre o ciclo hidrológico são drásticas, pois o aumento da temperatura do ar ocasiona o aumento da evaporação e da evapotranspiração, acarretando aumento da deficiência hídrica (Nobre et al., 2011). De fato, há essa tendência à desertificação na porção semiárida, até o final do século XXI, como consequência do aumento da temperatura do ar e da redução das chuvas, com a prática agrícola de sequeiro de alto risco, ainda mais marginal (Lacerda et al., 2010). Portanto, o conhecimento da disponibilidade hídrica é importante para quantificar os impactos da mudança do clima, viabilizando a adaptação. A disponibilidade hídrica está associada a diversos setores econômicos, fazendo com que eventuais alterações climáticas aumentem as pressões antrópicas sobre o uso sustentável da água (Bates et al., 2008).

iii) Fator 3 (F3): variáveis originais (VVM e VVMM) com as mais altas correlações "cargas fatoriais com a F3

O terceiro fator (F3) explica 16,38\% da variância total dos dados e possui pesos positivos e relacionados com as variáveis VVM e VVMM, sendo estas dependentes do relevo de uma determinada região, e o vento, tanto próximo à superfície terrestre, quanto em níveis mais elevados, tem influência direta no tempo e no clima, como erosão do solo, precipitação, formação de dunas, dispersão de sementes e poluentes. Portanto, este fator corresponde à circulação do ar atmosférico, sugerindo, um "Índice da circulação do ar" o qual determina o clima de qualquer região. 
A circulação atmosférica redistribui calor por todo o globo terrestre, podendo ser descrita por variáveis que caracterizam sua condição energética, não é homogênea, provocando variações nos elementos climáticos. O vento está associado às variações da pressão atmosférica, onde o movimento do ar, ocasionado pela associação entre a energia solar e a rotação planetária, a diferença de temperatura e de pressão entre dois locais, provocam um movimento horizontal (Oliveira et al., 2011).

iv) Fator 4 (F4): variáveis originais (PNMM, INS e NEBM) com as mais altas correlações "cargas fatoriais com a F4

$\mathrm{O}$ quarto fator (F4) explica apenas $12,57 \%$ da variância total dos dados e possui cargas fatoriais positivas para PNMM e NEBM, e negativa para INS. A pressão atmosférica apresenta variações conforme as altitudes e as temperaturas; a nebulosidade e a insolação estão relacionadas à variação na intensidade da radiação solar incidente na superfície, em razão da composição da atmosfera, em função da presença de nuvens, de poeira, de poluição e outros. Portanto, este fator está associado à altitude, à radiação e à presença de nuvens na atmosfera; pode-se interpretá-la como um "Índice da distribuição de nebulosidade atmosférica". As maiores cargas fatoriais positivas nesta componente são as varáveis PNMM $(0,777)$ e NEBM (0,497). A carga fatorial da variável insolação negativa $(-0,683)$ mostra que a precipitação tende a acompanhar o grau de nebulosidade, enquanto a insolação apresenta tendência inversa a de ambos.

A insolação recebida em um determinado local da superfície terrestre pode variar, e um dos fatores responsáveis por essa variação é a nebulosidade (Silva, 2011). Naturalmente que, em um dia nublado, a intensidade da radiação solar será menor e, consequentemente, o desempenho do módulo será prejudicado. Ocorre o contrário em dia claro ou com céu sem nuvens (Marques et al., 2000; Campos \& Alcantara, 2016).

A pressão atmosférica apresenta variações conforme as altitudes e as temperaturas. Sua manifestação está diretamente relacionada à força da gravidade e à influência que essa realiza sobre as moléculas gasosas que compõem a atmosfera. Assim, a pressão atmosférica mostra variações conforme as altitudes e as condições de temperatura do ar. Quanto maior a altitude de um dado relevo, isto é, quanto mais elevado ele estiver em relação ao nível do mar, menor será a pressão atmosférica (Bottecchia, 2009).

Cabe salientar que a interpretação dos fatores é subjetiva na análise, ou seja, o nome dado aos fatores depende de um ponto de vista subjetivo, e que, em muitas situações, torna-se difícil esta interpretação. Embora para cada fator apresentado tenham sido utilizadas apenas as variáveis mais importantes na sua composição, todas as variáveis entram, com coeficientes maiores ou menores, na formação dos índices.

Conforme mencionado, a base da criação dos índices de mudanças climático multivariado proposto neste estudo está na Matriz de Componentes após a rotação (Mingoti, 2007). Essa matriz apresenta os fatores e os coeficientes dos escores fatoriais (autovetores) normalizados maiores que um, em ordem decrescente de importância, na análise de cada fator, representam índices de mudanças climáticas.

Após obtenção, identificação dos fatores e determinação dos respectivos escores fatoriais padronizados (Equação 9) foi possível estudar o grau de mudanças climáticas, que se encontra associado a variabilidade das variáveis meteorológicas, e obtiveram-se os indicadores da Tabela 6.

Tabela 6. Análise descritiva, média e desvio-padrão (DP), carga fatorial (pi) das variáveis na ordem de maior peso e os índices climatológico multivariado (IC (Fij)) e a comunalidade (hi ${ }^{2}$ ) em cada fator, extraída em percentual. Fonte: Andrade et al. (2020).

\begin{tabular}{|c|c|c|c|c|c|c|}
\hline \multirow{2}{*}{ Fator } & \multirow{2}{*}{ Descrição da variável } & \multicolumn{2}{|c|}{ Descritiva } & \multirow{2}{*}{ pi } & \multirow{2}{*}{$\begin{array}{c}\text { IC(Fij) } \\
(\%)\end{array}$} & \multirow{2}{*}{$\mathbf{h i}^{2}$} \\
\hline & & Média & DP & & & \\
\hline \multirow{4}{*}{ F1 } & X1: TCM & 22,20 & 2,88 & 0,961 & \multirow{4}{*}{99,66} & 94,70 \\
\hline & X2: TMaM & 26,22 & 2,61 & 0,96 & & 94,60 \\
\hline & X3:TMiM & 19,24 & 2,77 & 0,935 & & 91,60 \\
\hline & X4: UR & 78,89 & 9,20 & $-0,913$ & & 87,90 \\
\hline \multirow{3}{*}{$\mathrm{F} 2$} & X5: PT & 29,82 & 20,56 & 0,959 & \multirow{3}{*}{56,07} & 97,20 \\
\hline & X6: NDP & 10,17 & 6,80 & 0,958 & & 96,70 \\
\hline & $\mathrm{X} 7: \mathrm{EVp}$ & 98,10 & 37,79 & $-0,83$ & & 90,10 \\
\hline F3 & X8: VVMM & 6,97 & 1,06 & 0,975 & 25,21 & 96,60 \\
\hline
\end{tabular}




\begin{tabular}{ccccccc} 
& X9: VVM & 3,49 & 0,66 & 0,813 & & 93,40 \\
& & & & & 86,80 \\
F4 & & 2,19 & 0,777 & & 91,80 \\
X10: PNMM & 154,04 & 50,89 & $-0,683$ & 21,11 & 80,10 \\
\hline & X11: INS & 6,98 & 1,94 & 0,497 & \\
\multicolumn{2}{l}{ TCM = Temperatura do ar compensada média $\left({ }^{\circ} \mathrm{C}\right) ; \mathrm{TMaM}=$ Temperatura do ar máxima média $\left({ }^{\circ} \mathrm{C}\right) ; \mathrm{TMiM}$}
\end{tabular}

$\mathrm{TCM}=$ Temperatura do ar compensada média $\left({ }^{\circ} \mathrm{C}\right) ; \mathrm{TMaM}=$ Temperatura do ar máxima média $\left({ }^{\circ} \mathrm{C}\right) ;$ TMiM $=$ Temperatura do ar mínima média $\left({ }^{\circ} \mathrm{C}\right) ; \mathrm{UR}=$ Umidade relativa média $(\%) ; \mathrm{PT}=$ precipitação total $(\mathrm{mm})$; $\mathrm{NDP}=$ Número de dias com precipitação $(\mathrm{qtd}) ; \mathrm{EVp}=$ Evaporação $(\mathrm{mm}) ; \mathrm{VVMM}=$ Velocidade do vento máxima média (mps); VVM = Velocidade do vento média (mps); PNMM = Pressão atmosférica ao nível do mar média (mbar); INS: Insolação total (horas) e NEBM = Nebulosidade média (décimos).

As comunalidades de cada variável são apresentadas na Tabela 6 e todas as comunalidades apresentaram um valor aceitável acima de 0,5, sugerido por Hair et al. (2010), o que sugere um bom ajuste do modelo nos nossos dados. Segundo Ribas \& Vieira (2011), as comunalidades indicam uma alta qualidade de explicação da variância de cada medição atribuída às componentes, ou seja, os valores encontrados para as comunalidades revelam que praticamente todas as variáveis têm sua variabilidade captada e representada pelos quatro fatores.

Outro aspecto importante na interpretação das comunalidades é entender quais as variáveis que mais impactam no modelo fatorial. Os maiores valores pertencem à precipitação total (PT) e ao número de dias com precipitação (NDP), estas são as variáveis que apresentam as maiores comunalidades, indicando que elas são as variáveis de maior importância para o estudo da variabilidade espacial de variáveis climática local, pois apresentam a maior porção da variância compartilhada com todas as outras variáveis consideradas.

Com base na Tabela 6, pode-se constatar que o fator F1 possui o menor nível de influência no clima local, quanto ao comportamento da temperatura do ar compensada média (TCM), temperatura do ar mínima média (TMiM), temperatura do ar máxima média (TMaM) e umidade relativa média (UR), correspondendo ao valor do índice climatológico multivariado de $99,66 \%$, indicando que essas as variáveis são as de maior importância nesse estudo quanto à de detecção de ocorrência de mudança climática local. Isto é, as distribuições espacial e temporal das variáveis TCM, TMiM, TMaM e UR têm menor peso ou contribuição na mudança do clima em Garanhuns. Isso se deve, possivelmente, pelo fato de que o clima desta região tem um sinal claro de variabilidade interanual associado às variações de temperatura do ar. No caso das regiões Norte e Nordeste do Brasil (NEB), vários estudos têm demonstrado que a precipitação tem também um forte acoplamento com as variações de temperatura do ar (Lacerda, 2015b).
No entanto, o fator $\mathrm{F} 4$ possui o menor índice de mudança climática, que é de $21,11 \%$, onde as varáveis que menos contribuem para determinar a ocorrência de mudança climática local são Pressão Atmosférica ao Nível do Mar Média (PNMM), Insolação Total (INS) e Nebulosidade Média (NEBM). De acordo com esses resultados, foram observadas evidências de mudanças locais no comportamento da Precipitação Total (PT), do Número de Dias com Precipitação (NDP) e Evaporação (EVp), correspondendo ao segundo maior valor do índice climatológico multivariado de 56,07\%, uma contribuição para determinar a ocorrência de mudança climática do município de Garanhuns.

Uma das consequências do aquecimento global na agricultura é o aumento da taxa de evapotranspiração das plantas. $\mathrm{O}$ aumento dessa taxa tende a ser especialmente prejudicial às atividades agrícolas, pois reduziria a água disponível para as plantas justamente na profundidade do solo onde estão as raízes, que, no caso das culturas anuais, são pouco profundas. Em regiões como o Nordeste do Brasil, principalmente no semiárido, a precipitação e a evaporação são variáveis determinantes das condições do clima local, bem como da sua variabilidade e mudança em longo prazo (Santos et al., 2009). Grande parte dos pesquisadores acredita que as alterações climáticas que vêm acontecendo são consequência da atividade antrópica, especificamente do aumento na emissão de Gases do Efeito Estufa (GEE) na atmosfera; outros acreditam que essas alterações climáticas se devem à variabilidade climática natural do planeta, colocando em dúvida as afirmações dos Relatórios de Avaliação do IPCC (2014). Apesar dessas incertezas referentes à influência do homem no clima, a grande maioria dos pesquisadores tem demonstrado que, nas últimas décadas, a atmosfera tem, de fato, mostrando um processo de variabilidade climática, com maior tendência de aquecimento.

De acordo com Cervi (2017), índices têm sua importância visto que é muito mais eficiente monitorar diversas variáveis através de uma única 
e não através de várias medidas. A criação destes índices, conforme Figueiredo Filho et al. (2013), pode ser realizada por meio da utilização da análise fatorial, quando há um grande número de variáveis necessárias para obtê-los, em que todas as variáveis são importantes. Por convenção, um índice é isento de dimensão, expresso na forma percentual, com dois dígitos e sem sinal indicativo (Milone, 2004; Bussab \& Morettin, 2010; Ayres, 2012).

\section{Análise de agrupamento hierárquico (AAH)}

A análise de agrupamento hierárquico foi aplicada à matriz dos escores das quatro componentes principais rotacionadas gerando um dendrograma que fornece uma visão bidimensional de $91,79 \%$ da informação estatística do sistema. Para sua construção não houve necessidade de pré-tratamento dos dados. Utilizou-se como medida de dissimilaridade o quadrado da distância euclidiana ao quadrado e, para a delimitação ou a formação dos grupos foi utilizado o método da distância média entre grupos. Essa análise confirmou a separação das 13 variáveis meteorológicas, em ordem dos escores do índice de mudança climática multivariado por grupo de contribuição e de homogeneidade, para delinear padrões de variações do clima local, sendo formados cinco grupos a um nível de distância euclidiana quadrática de 11, pelo método de agrupamento de ligação entre grupos (Figura 2).

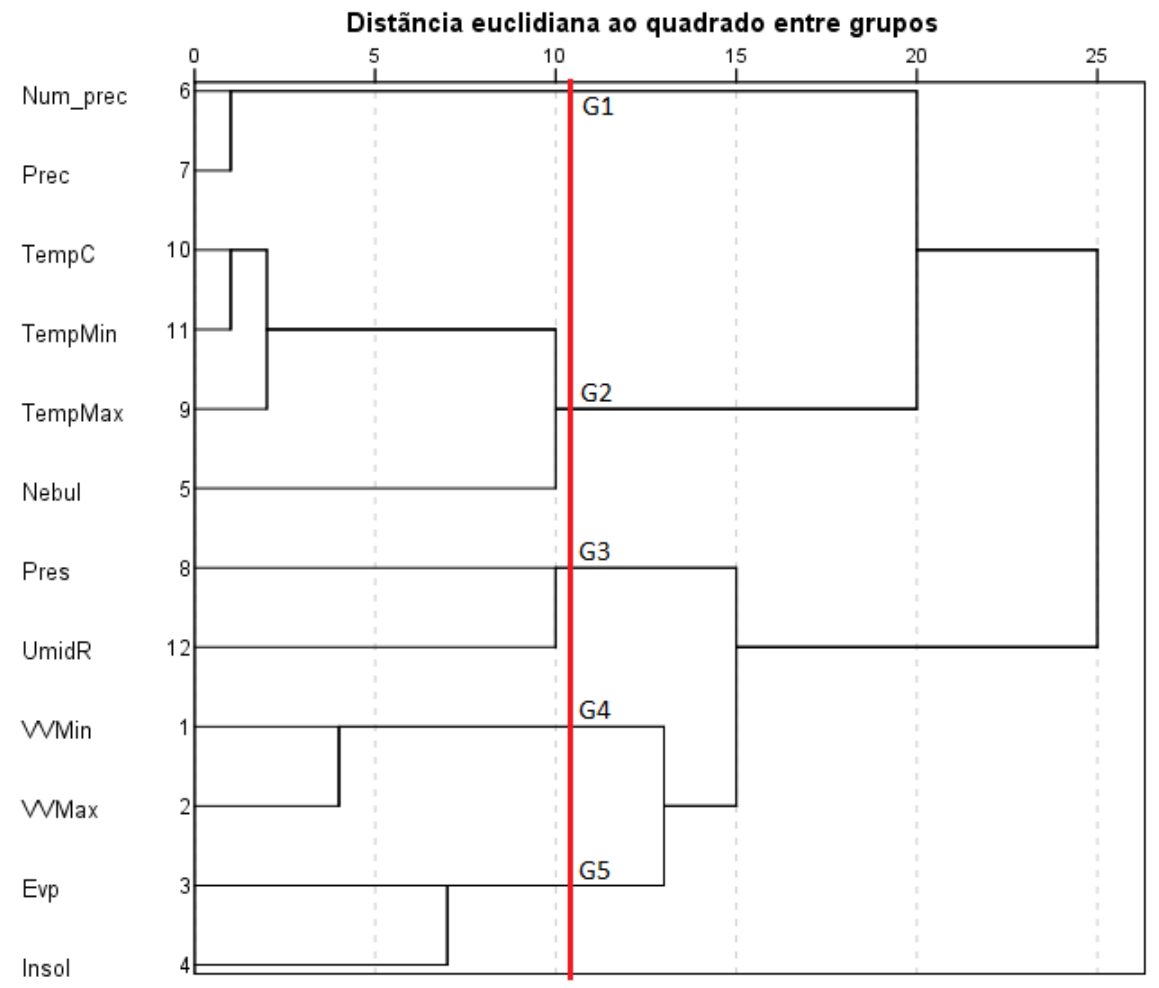

Figura 2. Dendrograma de agrupamento das variáveis meteorológicas da série histórica de 57 anos, no período de janeiro de 1963 a maio de 2019, do município de Garanhuns/PE, Brasil. Fonte: Fonte: Andrade et al. (2020).

No dendrograma mostrado na Figura 2 destacam-se cinco grupos. Existem duas variáveis meteorológicas nos grupos G1, G3, G4 e G5, enquanto no $\mathrm{G} 2$, s maior grupo, existem quatro variáveis. No grupo G1 têm-se as variáveis nebulosidade média (NEBM) e número de dias com precipitação (NDP), com relação inversa na formulação das mudanças do clima regional, pois constituem a porção semiárida da região Nordeste do Brasil, particularmente vulnerável às variações climáticas. A periodicidade das secas compromete, principalmente, a agricultura de subsistência que, na maior parte, se constitui de plantios em sistema de sequeiro (agricultura que não utiliza irrigação), principalmente no município de Garanhuns, onde é fortemente dependente da precipitação pluviométrica, e, consequentemente, as suas variações provocam prejuízos econômicos e sociais à população do município. Assim como em todo o Estado de Pernambuco, Garanhuns apresenta variações temporais e espaciais no regime de chuvas associada a mudanças climáticas com característica própria diferente dos demais regimes do NEB, apresentando uma grande variabilidade interanual.

O maior grupo G2 mostra a temperatura do ar compensada média (TCM), a temperatura do 
ar mínima média (TMiM), a temperatura do ar máxima média (TMaM) e a nebulosidade média (NEBM) são as variáveis meteorológicas que apresentam as maiores influências no clima da região. Esse grupo apresenta uma maior relação com as mudanças climáticas, com a contribuindo com a influência dos fatores naturais e de natureza antrópica os quais exercem alterações climáticas, ou seja, provocadas pela ação humana, provenientes das atividades industriais que liberam gases poluentes e de efeito estufa para a atmosfera, principalmente de dióxido de carbono, causando uma variabilidade dos elementos que compõem o clima.

Mudanças na temperatura do ar têm implicações no aquecimento global. Este fenômeno, gerado pelo aumento da poluição do ar, tem provocado o derretimento de gelo das calotas polares e o aumento no nível de água dos oceanos (IPCC, 2014). Espera-se que as mudanças climáticas alterem a temperatura média e os valores da precipitação, aumentando a variabilidade dos eventos, que poderão causar inundações e secas mais intensas e frequentes.

Lacerda et al. (2015a) afirmam que a frequência e a persistência das secas deverão ser uma das consequências do aquecimento global.

Segundo o Relatório do Painel Brasileiro de Mudanças Climáticas (PBMC, 2013), é provável que o semiárido nordestino tenha sua precipitação reduzida em até $20 \%$ até 2040 , com aumento de temperatura de até $1^{\circ} \mathrm{C}$. Este relatório também indica que todo o Brasil deverá ficar ao menos $3^{\circ} \mathrm{C}$ mais quente até o fim do século, as precipitações aumentariam em 30\% nas regiões Sul e Sudeste e diminuiriam em até $40 \%$ nas regiões Norte e Nordeste.

O grupo G3 é formado pelas variáveis, pressão atmosférica ao nível do mar média (PNMM) e umidade relativa média (UR). A umidade do ar é um dos elementos que compõe o clima d'água contido na atmosfera (Medeiros et al., 2006). Este vapor d'água é medido em índices: umidade absoluta, umidade relativa, temperatura do ponto de orvalho e pressão de vapor saturado. A medida mais conhecida é a umidade relativa devido à facilidade de obtenção dos dados, além de indicar o grau de saturação do ar (Varejão-Silva, 2006). Sendo influenciada por outros elementos que também compõem o clima como, precipitação pluvial e temperatura do ar, a umidade relativa do ar aumenta quando a temperatura diminui, e vice-versa (Varejão-Silva, 2006).

O grupo G4 é formado pelas variáveis velocidade do vento média (VVM) e a velocidade do vento máxima média (VVMM). Este grupo apresenta variável física influenciadora das condições climáticas. O Quinto Relatório do Painel Intergovernamental sobre Mudanças do Clima (IPCC, 2014), divulgado em outubro de 2018, indica alterações de deslocamento de ar na atmosfera, provadas pelas mudanças de temperatura nos continentes e oceanos, que podem mudar a distribuição das zonas de alta e baixa pressão atmosférica e causar a redução das chuvas.

O grupo G5 é formado apenas pelas variáveis Evaporação (EVp) e Insolação total (INS), as quais apresentaram as maiores similaridades deste grupo, pois apresentaram os maiores valores de distância, como pode ser observado no dendrograma (Figura 2). O vapor de água presente na atmosfera desempenha um papel importante na formação das nuvens, na sua duração, na quantidade de radiação solar que pode refletir, no tipo e local de precipitação que é gerado, e assim por diante. De acordo com Silva (2011), a insolação recebida em um determinado local da superfície terrestre pode variar, e um dos fatores responsáveis por essa variação é a nebulosidade. As nuvens são, evidentemente, essenciais para o nosso clima; as concentrações e a composição das partículas podem realmente alterar o calendário e a localização dos padrões de precipitação tradicionais. As nuvens fazem com que ocorra uma variação na intensidade da radiação solar incidentes na superfície, e por conta dessa variação de radiação, explica-se a variabilidade de radiações incidente de uma região (Holanda et al., 2017; Campos \& Alcantara, 2016).

Por outro lado, as diferenças nos agrupamentos observadas nesse estudo, a heterogeneidade intergrupos, entre as variáveis metrológicas, está relacionada, em parte, à variabilidade interanual da Temperatura da Superfície do Mar (TSM) nos Oceanos Pacífico e Atlântico, nas micro e mesorregiões do Estado, bem como a verificação das anomalias da Zona de Convergência Intertropical; na pós-estação, às Ondas de Leste e às formação de vórtices ciclônicos de ar superior e também na influência de frentes frias que vêm até a Bahia e, eventualmente, induzem chuvas no Estado de Pernambuco, como principal variável física influenciadora das condições climáticas e dos impactos das alterações climáticas (Germano, 2016).

A Organização Meteorológica Mundial (OMM) criou um grupo de trabalho que elaborou índices de detecção de mudanças climáticas. Ao todo, foram definidos 27 índices de detecção de mudanças climáticas, dos quais, 11 são 
decorrentes da precipitação e 16 da temperatura do ar (Santos et al., 2006). No Brasil, diversos estudos também têm se baseado na estimativa dos índices de detecção de mudanças climáticas da OMM para a avaliação das mudanças climáticas locais (Almeida et al., 2010). Porém, a maior parte destes se concentra na região Sul e Sudeste do país, visto que essas regiões apresentam maior quantidade de estações e séries com melhor qualidade e intervalo de dados.

A região Nordeste possui também como característica natural um alto potencial para a evaporação da água, em função da enorme disponibilidade de energia solar e altas temperaturas. Aumentos da temperatura do ar, associados à mudança de clima, decorrentes do aquecimento global, independentemente do padrão de chuva, são suficientes para causar maior evaporação dos açudes e reservatórios e aumento da demanda evaporativa pelas plantas. A menos que haja um aumento de chuvas, a água se tornará mais escassa (Marengo et al., 2011; Lacerda et al., 2015a).

Estudos dos impactos das mudanças climáticas na estabilidade dos biomas brasileiros (Nobre, 2011; Lacerda et al., 2015a) revelam que o bioma Caatinga está entre os mais vulneráveis em um cenário de aumento das temperaturas globais, o que coloca a região em alerta, uma vez que os efeitos das mudanças climáticas representam fatores a mais em relação à pressão de origem antrópica à desertificação.

\section{Conclusões}

Conclui-se que o uso de técnicas multivariadas como a Análise Fatorial pode auxiliar na redução da dimensão dos dados, pois é possível agrupar variáveis mais correlacionadas, fazendo com que a perda das informações seja a menor possível. Com a aplicação da técnica Análise Fatorial, resumiu-se um conjunto de 13 variáveis meteorológicas influenciadoras das condições climáticas para quatro descritores estatísticos (fatores): "Indices das distribuições espacial e temporal da temperatura do ar e do teor de água na atmosférica", "Índice da circulação do ar - direção predominante do vento local".

Foi possível identificar as variáveis mais representativas na obtenção de índices de detecção de mudanças climáticas multivariado, considerando variáveis meteorológicas influenciadoras das condições climáticas para o município de Garanhuns/PE.

\section{Agradecimentos}

Agradecemos à Fundação de Amparo à Ciência e Tecnologia de Pernambuco (FACEPE) pelo financiamento de pesquisa, por meio do Programa Institucional de Bolsas de Iniciação Científica (PIBIC), pela concessão de bolsa de Iniciação Científica do discente Anízio Honorato Godoy Neto.

\section{Referências}

Almeida, R.; Rebello, E.; Ambrizzi, T. 2010. Variabilidade de eventos extremos e identificação de tendências climáticas no litoral Norte do Brasil. 2010. In: XVI Congresso Brasileiro de Meteorologia-CBM, Belém, PA, Brasil. pp. 1-6.

Andrade, A. R S.; Neto, A. H. G.; Cruz, A. F. S.; Andrade, E. K. P.; Santos, V. F.; Silva, T. N. P. 2018. Geoestatística aplicada à variabilidade espacial e padrões nas séries temporais da precipitação no Agreste pernambucano. 2018. Journal of Environmental Analysis and Progress, 3, 1, 126-145.

Ayres, M. 2012. Elementos de bioestatística: a seiva do açaizeiro. 2. Ed. Belém: Super cores, 588 p.

Barroso, L. P.; Artes, R. 2003. Análise multivariada. São Paulo: IME, USP, 155p.

Bates, B. C.; Kundzewicz, Z. W.; Wu, S.; Palutikof, J. P. 2008. Climate change and water. Artigo Técnico do Painel Intergovernamental sobre Mudanças Climáticas, Genebra: Secretariado do IPCC, 210p.

Bezerra, A. C. N.; Pezzi, L. P.; Kayano, M. 2008. Esquema estatístico de combinação e correção de previsões climáticas ECCOCLIM. Revista Brasileira de Meteorologia, 23, 3, 347-359.

Boscarioli, C. 2008. Análise de Agrupamentos baseada na topologia dos dados e em mapas auto-organizáveis. Tese de Doutorado, Escola Politécnica da Universidade de São Paulo. São Paulo, Brasil. 118p.

Bottecchia, O. L. 2009. A fórmula barométrica como instrumento de ensino em química. Quim. Nova, 32, 7, 1965-1970.

Bussab, W. de O.; Morettin, P. A. 2010.Estatística básica. 6.ed. São Paulo: Ed. Saraiva, 540p.

Camelo, H. N.; Lucio, P. S.; Junior, J. B. V. L.; Carvalho, P. C. M. 2017. Métodos de Previsão de Séries Temporais e Modelagem Híbrida ambos Aplicados em Médias Mensais de Velocidade do Vento para Regiões do Nordeste do Brasil. Revista Brasileira de Meteorologia, 32, 4, 565-574.

Camelo, H. N.; Carvalho, P. C. M.; Junior, J. B. V. L.; Accioly Filho, J. B. P. 2008. Análise estatística da velocidade de vento do estado 
do Ceará. Revista Tecnologia, 29, 2, 211223.

Campos, M. M.; Alcantara, L. D. 2016. Interpretação dos efeitos de tempo nublado e chuvoso sobre a radiação solar em Belém/PA para uso em sistemas fotovoltaicos. Revista Brasileira de Meteorologia, 31, 4, 570-579.

Cervi, U. E. 2017. Métodos quantitativos para iniciantes em ciência política, Curitiba, PR, Crop/UFRPR, 256p.

Chen, W.; Baghdasaryan, L.; Buranathiti, T.; Cao, J. 2003. Model Validation via Uncertainty Propagation and Data Transformations. Journal AIAA, 1, 1-16.

Cordeiro, R. F.; Andrade, B. L. S.; Pinto, R. L. M. 2017. Aplicação da Técnica Estatística Multivariada Análise Fatorial para o Serviço de Transporte da Cidade de João Monlevade. Anais VI Congresso brasileiro de Engenharia de Produção-CBEP, Ponta Grossa, PR, Brasil. pp. 11-15.

Corrar, L. J.; Paulo, E.; Dias Filho, J. M. 2009. Análise multivariada para os cursos de administração, ciências contábeis e economia. São Paulo: Editora Atlas, 541p.

Costa, M. B. S. F.; Mallmann, D. L. B.; Pontes, P. M.; Araujo, M. 2010. Vulnerability and impacts related to the rising sea level in the Metropolitan Center of Recife, Northeast Brazil. Pan-American Journal of Aquatic Sciences, 5, 2, 341-349.

Coutinho, M. L.; Nascimento, M. G.; Coutinho, M. D. L.; Souza, E. P. 2006. Simulação de precipitação no NEB através de testes de Esquemas de Convecção do BRAMS. Anais do XIV Congresso Brasileiro de Meterorologia-CBMet, Florianópolis, SC, Brasil. pp. 20-25.

Fava, J. L.; Velicer, W. F. 1992. The effects of over extraction on factor and component analysis. Multivariate Behavioral Research, 27, 3, 387-415.

Fávero, L. P.; Belfiore, P.; Silva, F. L.; Chan, B. L. 2009. Análise de dados: modelagem multivariada para tomada de decisões. Rio de Janeiro: Editora Elsevier, 210p.

Field, A. 2009. Descobrindo a estatística usando o SPSS. Tradução de Lorí Viali. Porto Alegre, RS, $2^{a}$. ed., Editora Artmed, 687p.

Figueiredo Filho, D. B.; Paranhos, R.; Rocha, E. C.; Silva Júnior, J. A.; Maia, R. G. 2013. Análise de componentes principais para construção de indicadores sociais. Rev. Bras. Biom., 31, 1, 61-78.

Freire, J. L. M.; Freitas, S. R.; Coelho, C. A. S. 2015. Calibração do modelo regional BRAMS para a previsão de eventos climáticos extremos. Rev. Bras. Meteorol., $30,2,70-81$.

Freitas, J. C. 2009. Análise de agrupamentos na identificação de regiões homogêneas de índices climáticos no estado da Paraíba - PB. Dissertação de Mestrado, Universidade Federal de Campina Grande. Campina Grande, PB, 75p.

Freitas, S. M.; Prata, B. A. 2007. Uma nova abordagem para a análise de agrupamento com uma aplicação em agronomia. In: Reunião Anual da RBRAS e Simpósio de Estatística Aplicada a Experimentação Agronômica, 2007, Santa Maria, RS. Anais: $52^{\mathrm{a}}$ Reunião da RBRAS e $12^{\circ}$ SEAGRO. Santa Maria, RS, Brasil. pp.1-5.

Freitas, S. R.; Rodrigues, L. F.; Longo, K. M.; Panetta, J. 2012. Impact of a monotonic advection scheme with low numerical diffusion on transport modeling of emissions from biomass burning. Journal of Advances in Modeling Earth Systems, 4, 210-323.

Germano, L. S. 2016. Aspectos sinóticos de vórtices ciclônico em altos níveis que provocaram precipitação extremas nas cidades de recife, Maceió e Aracaju no período de 2011 a 2015: Estudo de caso. Dissertação de Mestrado, Universidade Federal de Alagoas. Maceió, AL, Brasil, 87p.

Giongo, V. 2011. Balanço de Carbono no semiárido brasileiro: Perspectivas e Desafios em: Desertificação e Mudanças Climáticas no Semiárido Brasileiro. Editores: R. C. C. Lima, A. M. B. Cavalcante e A. M. P. Marin, Instituto Nacional do Semiárido, INSA, pp. 115-130.

Gneiting, T.; Raftery, A. E. 2005. Weather Forecasting with Ensemble Methods. Science, 310, 5746, 248-249.

Gois, G.; Souza, J. L.; Silva, P. R. T.; Júnior. F. O. 2005. Caracterização da desertificação no estado de Alagoas utilizando variáveis climáticas. Revista Brasileira de Meteorologia, 20, 3, 301-314.

Guedes, R. V. S.; Lima, F. J. L.; Amanajás, J. C.; Braga, C. C. 2010. Análise em componentes principais da precipitação pluvial no estado do piauí e agrupamento pelo método de Ward. Revista de Geografia. 27, 1, 218-232, 2010.

Hair, J.; Black, W.; Babin, B.; Anderson, R. 2010. Multivariate Data Analysis. 7. ed. Pearson Prentice Hall, 593p.

Holanda, R. M.; Medeiros, R. M.; Lorena, E. M. G.; Kozmhinsky, M.; Silva, V. P.; Moraes, A. S. 2017. Flutuação da insolação e nebulosidade no município de Caruaru - PE, 
Brasil. Anais III Workshop Internacional sobre água no Semiriádo Brasileiro, Campina Grande, PB, Brasil. pp. 1-5.

INMET. Instituto Nacional de Meteorologia. 2019. Disponível em: http://www.inmet.gov.br/portal/index.php?r =clima/normaisclimatologicas. Acessado em: 20 de julho de 2019.

IPCC. Intergovernmental Panel on Climate Change: Climate Change 2014: Synthesis Report. 2014. Contribution of Working Groups I, II and III to the Fifth Assessment Report of the Intergovernmental Panel on Climate Change [Core Writing Team, R.K. Pachauri and L.A. Meyer (eds.)]. IPCC, Geneva, Switzerland, 151p.

Kayano, M. T.; Andreoli, R. V.; Souza, R. A. F. 2011. Evolving anomalous SST patterns leading to ENSO extremes: relations between the tropical Pacific and Atlantic Oceans and the influence on the South American rainfall. International Journal of Climatology, 31, 1119-1134.

Kundzewicz, Z. W.; Mata, L. J.; Arnell, N.; Doel, P. 2007. Freshwater resources and their management. Climate Change 2007: Impacts, Adaptation and Vulnerability. Contribution of Working Group II to the Fourth Assessment Report of the Intergovernmental Panel on Climate Change (IPCC) [Parry, M.L. (Eds.)], Cambridge, UK, New York, NY: Cambridge University Press., pp.173210.

Kurnaz, M. L. 2004. Detrended fluctuation analysis as a statistical tool to monitor the climate. Journal of Statistical Mechanics: Theory and Experiment, 1-11, 2004.

Lacerda, F. F. 2015. Tendências de temperatura e precipitação e cenários de mudanças climáticas de longo prazo no nordeste do Brasil e em ilhas oceânicas. Tese de Doutorado, Universidade Federal de Pernambuco, Recife, 109p.

Lacerda, F. F.; Nobre, P.; Lopes, G. M. B. O. 2015a. Clima e suas Alterações em Pernambuco. In: Mudanças Climáticas e Resiliência de Cidades / organizadores Fátima Furtado, Luiz Priori, Ednéa Alcântara - Recife: Pickimagem, pp. 61-72.

Lacerda, F. F.; Nobre, P.; Sobral, M. C.; Lopes, G. M. B.; Chan, C. S.; Brito, E. 2015b. Long term climate trends over Nordeste Brazil and Cape Verde. Journal of Earth Science \& Climatic Change. pp. 112-123.

Lacerda, F. F.; Silva Júnior, H. D. da; Assad, E. D.; Assis, J. M. O.; Moura, M. S. B. 2010. Extremos e variabilidade climática no
Nordeste brasileiro e em Pernambuco. In: Mudanças Climáticas e Impactos Ambientais. Recife, PE: Editora Universitária da UFPE, Recife. 342p.

Longo, K. M.; Freitas, S. R.; Dias, M. A. S.; Dias, P. L. S. 2006. Numerical modeling of the biomass-burning aerosol direct radiative effects on the thermodynamics structure of the atmosphere and convective precipitation. In: International Conference on Southern Hemisphere Meteorology and OceanographyICSHMO, pp. 283-289.

Manly, B. J. F. 2008. Métodos estatísticos multivariados: uma introdução. Tradução de Sara Ianda Carmona. 3. ed. Porto Alegre: Bookman, 229p.

Marengo, J. A.; Alves, L. M.; Bezerra, E. A.; Lacerda, F. F. 2011. Variabilidade e mudanças climáticas no semiárido brasileiro. In: Variabilidade e mudanças climáticas no semiárido brasileiro. $1^{\text {a }}$. ed., Campina Grande, PB. Instituto Nacional do Semiárido, 1, pp. 383-416.

Marques, K.; Pereira, T. P.; Assis, S. V. 2000. Análise do comportamento mensal do Índice de Limpidez. In: XI Congresso Brasileiro de Meteorologia, edição XI. Anais..., Rio de Janeiro, pp. 174-181.

Maxwell. Um procedimento inferencial para análise fatorial utilizando as técnicas bootstrap e jackknife: construção de intervalo de confiança e testes de hipóteses. Disponível em:http://www.maxwell.vrac.pucrio.br/Busca . Acesso em: 02 julho 2019.

Medeiros, R. M.; Sousa, F. A. S.; Filho, M. F. G.; Francisco, P. R M. 2013. Variabilidade da umidade relativa do ar e da temperatura máxima na bacia hidrográfica do rio Uruçuí Preto. Revista Educação Agrícola Superior Associação Brasileira de Educação Agrícola Superior, 28, 1, 4-50.

Meireles A. C. M.; Oliveira L. J. 2011. Sustentabilidade do modelo agrícola da bacia do riacho Faé. Revista Ciência Agronômica, 42, 1, 84-91.

Melo, C. O.; Parré, J. L. 2007. Índice de desenvolvimento rural dos municípios paranaenses: determinantes e hierarquização. Revista de Economia e Sociologia Rural. Rio de Janeiro, 45, 2, 329-365.

Milone, G. 2004. Estatística Geral e Aplicada. São Paulo, Brasil. Pioneira Thomson Learning, 484p.

Mingoti, S. A. 2077. Análise de dados através de métodos de estatística multivariada: uma abordagem aplicada. Belo Horizonte: Editora UFMG, 297p. 
Molion, L. C. B.; Bernardo, S. O. 2002. Uma revisão da dinâmica das chuvas no nordeste brasileiro. Revista Brasileira de Meteorologia, 17, 1-10.

Monteiro, V. P.; Pinheiro, J. C. 2004. Critério para implantação de tecnologias de suprimentos de água potável em municípios cearenses afetados pelo alto teor de sal. Revista de Economia e Sociologia Rural, 42, 2, 365-387.

Nobre, P. 2011. Mudanças Climáticas e desertificação: os desafios para o Estado Brasileiro. In: Desertificação e Mudanças Climáticas no Semiárido Brasileiro. Editores: R. C. C. Lima, A. M. B. Cavalcante e A. M. P. Marin, Instituto Nacional do Semiárido INSA, pp. 25-35.

Oliveira, J. L.; Costa, A. A. 2011. Estudo de variabilidade do vento em escala sazonal sobre o nordeste. Revista Brasileira de Meteorologia, 26, 1, 53-66.

PBMC. Painel Brasileiro De Mudanças Climáticas. 2013. Contribuição do Grupo de Trabalho 1 ao Primeiro Relatório de Avaliação Nacional do Painel Brasileiro de Mudanças Climáticas. Sumário Executivo GT1. PBMC, Rio de Janeiro, RJ, Brasil, 24p.

Pernambuco. 2006. Secretaria de Ciência, Tecnologia e Meio Ambiente. Atlas de Bacias Hidrográficas. Recife, PE, Brasil, $103 p$.

Pezzi, L. P.; Ubarana, V.; Repelli, C. 2000. Desempenho e previsões de um modelo regional estatístico para a região sul do
Brasil. Brazilian Journal of Geophysics, 18, 2, 75-86.

Ribas, J. R.; Vieira, P. R. C. 2011. Análise multivariada com o uso do SPSS. Rio de Janeiro: Editora Ciência Moderna, 272p.

Santos, C. A. C. 2006. Estimativa e Tendências de Índices de Detecção de Mudanças Climáticas com base na precipitação diária no Rio Grande do Norte e na Paraíba. Dissertação de Mestrado, Universidade Federal de Campina Grande, Campina Grande, PB, 98p.

Santos, C. A. C.; Brito, J. I. B.; Rao, T. V. R.; Menezes, H. E. A. 2009.Tendências dos índices de precipitação no Estado do Ceará. Revista Brasileira de Meteorologia, 24, 1, 3947.

Silva, N. C. N.; Ferreira, W. L.; Cirillo, M. A.; Scalon, J. D. 2014. O uso da análise fatorial na descrição e identificação dos perfis característicos de municípios de Minas Gerais. Rev. Bras. Biom., 32, 2, 201-215.

Silva, V. A. M. 2011. Influência da cobertura do céu na estimativa da radiação solar utilizando modelo digital de elevação. Dissertação de Mestrado, Universidade Federal de Mato Grosso, Cuiabá, MG, 73p.

Varejão-Silva, M. A. 2006. Meteorologia e climatologia. Recife: Versão Digital, 155p.

Varejão-Silva, M. A. 2000. Estimativa da temperatura do ar à superfície. Brasília, DF: INMET, 98p. 